\title{
A non-compact formulation for job-shop scheduling problems in traffic management
}

\author{
Leonardo Lamorgese $^{*} \quad$ Carlo Mannino ${ }^{\dagger}$
}

\begin{abstract}
A central problem in traffic management is that of scheduling the movements of vehicles so as to minimize the cost of the schedule. It arises in important applications such as train timetabling, rescheduling, delay and disruption management, airplane surface routing, runway scheduling, air traffic control and more. This problem can be modeled as a job-shop scheduling problem. We introduce a new MILP formulation for job-shop scheduling which is alternative to classical approaches, namely big- $M$ and time-indexed formulations. It does not make use of artificially large coefficients and its constraints correspond to basic graph structures, such as paths, cycles and trees. The new formulation can be obtained by strengthening and lifting the constraints of a classical Benders' reformulation. Tests on a large set of real-life instances from train rescheduling show the new approach performs on average better than our previous approaches based on big$M$ formulations and particularly better on a class of instances with non-convex costs very common in the practice.
\end{abstract}

\section{Introduction}

A central problem in transportation is that of routing and scheduling the movements of vehicles so as to minimize the cost of the schedule. It arises, for instance, in train timetabling [10], rescheduling (or dispatching) [9], delay and disruption management [12], runway scheduling [6], airplane surface routing [16], and many more. These are both online (real-time) and off-line problems. For instance, train timetabling amounts to finding a long term (official) schedule of all trains movements, whereas in its online version, train rescheduling, the timetable is modified in real-time in order to react and recover from unexpected deviations from the plan. In both cases, the ability to find good or optimal schedules in short computing time is crucial: for real-time problems, because decisions must be taken and implemented in a few seconds; for off-line problems, because

*OptRail, Rome, Italy, e-mail: leonardo.lamorgese@optrail.com

†Sintef Digital and University of Oslo, e-mail: carlo.mannino@sintef.no 
the scheduling problem must generally be solved repeatedly in the overall solution framework.

For the fixed routing case, the problem boils down to finding a minimum cost conflict-free schedule, i.e. a schedule where potential conflicts are prevented by a correct timing of the vehicles on the shared resources. Namely, whenever two vehicles want to access the same, non-sharable resource - e.g. a runway or a station platform - a decision about who goes first [28] must be taken. In [22] this problem is shown to belong to the class of job-shop scheduling problems with blocking and no-wait constraints, a class which generalizes canonical job-shop scheduling (see [26]).

This decision is modeled as a disjunction of two linear constraints in the scheduling variables $[3,5]$. So, a basic mathematical representation of the scheduling problem involves continuous variables representing times, (time-precedence) linear constraints associated with single vehicles, and disjunctive (precedence) linear constraints associated with pairs of vehicles. In this paper we focus on this basic mathematical representation, which is the central building block for modelling and solving real-life scheduling problems. There are two major ways to linearize disjunctive formulations (see [27]), namely by means of big- $M$ formulations or by time-indexed formulations ${ }^{1}$. In big- $M$ formulations each disjunction is represented with two linear constraints and one (or two) binary variable(s), where the variables select which of the two constraints must be satisfied by the schedule (the other constraint becomes redundant). Although big- $M$ formulations are generally very compact, notoriously they tend to produce weak relaxations and consequently large search trees. Time-indexed formulations were introduced precisely to address this major drawback: the time horizon is subdivided into periods, with a binary variable for each original scheduling variable and each time period ([14]). Disjunctive constraints are expressed by cardinality constraints in these variables. Time-indexed formulations typically return much stronger bounds than big- $M$ formulations. However this comes at the cost of increasing the number of variables and constraints, at times dramatically, particularly for problems with large time horizons and fine time granularity (typically the case for traffic management problems in transportation). In our experience for train rescheduling [20], the trade-off is significantly in favour of big- $M$ formulations. Indeed in [20], for time-indexed formulations, the increase in computing time to solve the linear relaxation due to larger instance size is not even closely compensated by the reduced size of the search tree. For this reason, in the recent development of a master/slave approach to train rescheduling ([17, 18]) we resorted to the big- $M$ formulation for modeling and solving the master (job-shop scheduling) problem.

Despite the effectiveness of this approach, which led to successful real-life implementations, the "big- $M$ trap" (i.e. the intrinsic limitation of the approach) remained behind the corner. To speed up the solution of the subproblems and/or tackle larger instances required strengthening the big- $M$ formulation, which has been attempted for decades with little or no success. In very recent years, some authors have attempted to overcome this issue by exploring new paths. For instance, Bonami et al. [8] developed a

\footnotetext{
${ }^{1}$ See [8] for a discussion on how to handle generic indicator constraints
} 
new, general method borrowed from Non-Linear Programming. However, according to the figures presented in [8], the application to scheduling problems appears to lead only to small improvements. A different approach, explicitly designed for train rescheduling, was presented in [15]. The authors show this approach can solve some real-life instances in the very short running times dictated by real-time. However, the approach is heuristic, and the exact delayed row generation algorithm presented in $[17,18]$ seems to lead to larger speeds up (although computational results cannot be directly compared as they relate to different sets of proprietary instances).

In this paper we develop a new, non-compact formulation for the job-shop scheduling problem with separable, non-decreasing non-negative cost function [31]. This new formulation allows to overcome the limitations of previous models and avoid the big- $M$ trap (it does not make use of artificial large coefficients). The constraints in the formulation correspond to basic structures like paths, cycles and trees in an associated graph. We show how such constraints may be derived by strengthening and lifting from a classical Benders' reformulation [23]. We test the new approach on a large set of real-life instances from train rescheduling and show that it compares favorably with respect to the (effective) approach previously developed in [17, 18]. The average improvement in terms of computation time is small for convex costs, but becomes significant for the non-convex case (see Section 5). As mentioned, obtaining such speed-up is (clearly) particularly relevant in solving real-time traffic management problems (e.g. runway sequencing, train re-scheduling, etc.), but can also be important when attacking feasibility off-line problems, like the class of train timetabling problems presented in [19].

Summarizing, in $([17,18])$ we apply the so called Logic Benders' reformulation (see [11]) in order to decompose the original problem into a "line problem" (i.e. relative to the railway line) and a number of station problems (i.e. associated with each station), with the line problem being a big- $M$ formulation (of a scheduling problem). In this paper we only focus on the line problem defined in the above decomposition, and proceeding from the classic Benders' reformulation we show how to get rid of the big- $M$ constraints.

As mentioned earlier, our problem is a job-shop scheduling problem. Each vehicle is a job, each network resource (e.g. roads, crossings, waypoints, tracks) is a machine and the (sequence of) movements of each vehicle through the network are operations. Now, job-shop scheduling problems in traffic management have some common features for which the new approach is particularly suited. First, the number of disjunctions is limited, typically in the order of, or smaller, than the number of operations (whereas in principle this number could grow quadratically). Secondly, and perhaps more importantly, the objective function is associated with the schedule of few operations, sometimes even only one operation per job (i.e. the arrival time, or delay, of the vehicle at some specific target location). However, the approach developed in this paper can in principle be applied to a more general class of job-shop scheduling problems. 


\section{A big- $M$ formulation for the job-shop scheduling problem in traffic management}

In this section we formally introduce the job-shop scheduling problem considered in this paper. We then introduce a natural Mixed Integer Linear Program (MILP) for the problem. Finally we transform the natural formulation in a way which is suitable for the developments in the following sections.

The movement of a vehicle through a transportation network can be modeled as a sequence of atomic movements, corresponding to the occupation of a specific network resource. For example, a train in a railway network runs through a sequence of tracks; an airplane in the airdrome runs through a sequence of taxiways; a vehicle on a road through road segments, etc. The ordered sequence of atomic movements associated with a vehicle is called route. Let $O$ be the set of atomic movements of all vehicles. With every atomic movement $u \in O$ we associate the quantity $t_{u} \in \mathbb{R}_{+}$representing the time the vehicle starts atomic movement $u$ (i.e. the vehicle starts occupying the corresponding network resource). The vector $t \in \mathbb{R}_{+}^{|O|}$ is called schedule. If we consider two successive atomic movements $u, v \in O$ on the route of a vehicle, then we have that the schedule satisfies the time-precedence (linear) constraint

$$
t_{v}-t_{u} \geq \lambda_{u v}
$$

where $\lambda_{u v}$ is the minimum time necessary for the vehicle to complete the atomic movement $u$. Now, consider two distinct vehicles $A$ and $B$, both going through a non-sharable network resource $R_{1}$, as in Figure $1 .^{2}$

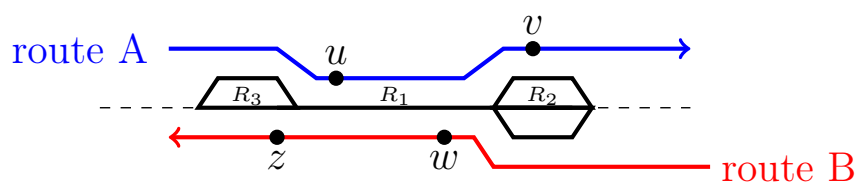

Figure 1: The routes of two trains running through the single track section $R_{1}$ in opposite directions.

Let $u \in O$ be the atomic movement on the route of $A$ corresponding to the occupation of resource $R_{1}$, and let $v$ be the next movement - corresponding to the occupation of a new resource $R_{2}$. Similarly, let $w$ be the movement corresponding to resource $R_{1}$ on the route of $B$ and let $z$ be the next movement (corresponding to a resource $R_{3}$ which may be distinct from $R_{2}$ ). Since the resource $R_{1}$ is non-sharable, either vehicle $A$ precedes $B$ on $R_{1}$ or viceversa. In the first case, $A$ enters the next resource $R_{2}$ before

\footnotetext{
${ }^{2}$ Note that here we consider a basic case of incompatible movements. More complicated cases can be easily handled in a similar fashion.
} 
$B$ enters $R_{1}$, that is $t_{w} \geq t_{v}$; in the second case $B$ enters $R_{3}$ before $A$ enters $R_{1}$, and we have $t_{u} \geq t_{z}$. Therefore, the schedule $t$ must satisfy the following disjunctive constraint:

$$
\left(t_{w}-t_{v} \geq 0\right) \bigvee\left(t_{u}-t_{z} \geq 0\right)
$$

Observe that the above disjunction consists of two terms, each term being a (linear) precedence constraint in the schedule variables ${ }^{3}$.

A job-shop scheduling problem in traffic management can be represented by a directed graph whose nodes correspond to atomic movements, whereas the edges correspond to linear precedence constraints and to disjunctions. In the graph of Figure 2 each disjunction is represented by a pair of directed edges (dotted edges in the picture), one for each term of the disjunction

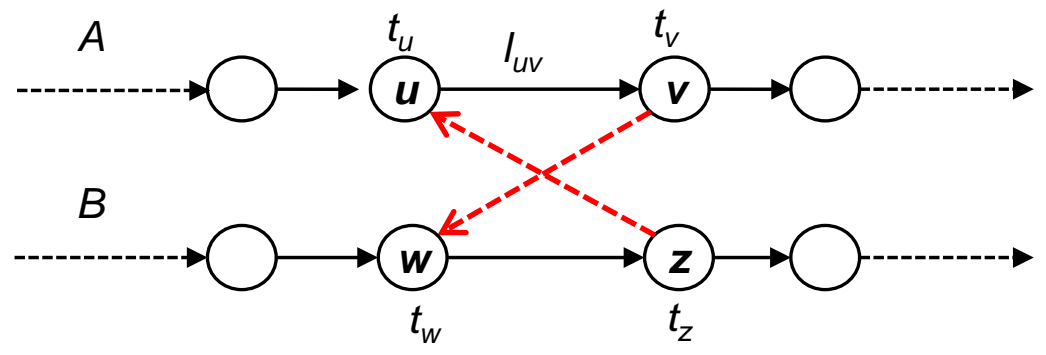

Figure 2: A directed graph representing a job-shop scheduling instance

We are now able to state the problem addressed in this paper:

Problem 2.1 Let $O$ be a set of atomic movements, let $t_{u}$ be the starting time of movement $u \in O$ and let $O^{2}=O \times O$ be the set of the ordered pairs of atomic movements. Let $A \subseteq O^{2}$ be a set of (indices of) linear precedence constraints with associated r.h.s. $\lambda \in \mathbb{R}_{+}^{|A|}$. Let $D \subseteq\left\{\{e, f\}: e, f \in O^{2}, e \neq f\right\}$ be the set of (indices of) disjunctive precedence constraints. Finally, let $c: \mathbb{R}_{+}^{|O|} \rightarrow \mathbb{R}$ be the cost of the schedule. Find a schedule $t \in \mathbb{R}_{+}^{|O|}$ satisfying all simple precedence constraint (1), for $(u, v) \in A$, and all disjunctive precedence constraints (2), for $\{(v, w),(z, u)\} \in D$, and such that $c(t)$ is minimized.

Next, we introduce the set $F=\left\{(u, v) \in O^{2}:\{(u, v),(w, z)\} \in D\right\}$ of the simple precedence constraints contained in one or more disjunctions of $D$. For the sake of simplicity, in the remainder of the paper we assume $F$ to be a simple set - the generalization is immediate.

\footnotetext{
${ }^{3}$ In principle, the r.h.s. of each term may be non-zero. We prefer here this version in order to simplify the following discussion
} 
Problem 2.1 can be easily represented as a Mixed Integer Program (MIP). For each $(u, v) \in F$ we introduce a binary variable $y_{u v}$, with $y_{u v}=1$ if the schedule satisfies $t_{v}-t_{u} \geq 0$. Then Problem 2.1 can be written as the following MIP:

$$
\begin{array}{lll}
\min & c(t) & \\
\text { s.t. } & & (u, v) \in A \\
\text { (i) } & t_{v}-t_{u} \geq \lambda_{u v}, & (u, v) \in F, \\
(i i) & t_{v}-t_{u} \geq M\left(y_{u v}-1\right) & \{(u, v),(w, z)\} \in D, \\
(i i i) & y_{u v}+y_{w z}=1, & u \in O, \\
(i v) \quad & t_{u} \geq 0, & \\
& t \in \mathbb{R}^{|O|} \quad y \in\{0,1\}^{|F|} &
\end{array}
$$

where $M$ is a suitably large constant and $\lambda_{u v} \geq 0$ for all $u v \in A$. Constraints (3.ii) and (3.iii) ensure that at least one simple precedence constraint in every disjunction is satisfied by $t$.

Program (3) is the "natural" formulation for our problem, adopted, in a form or another, by many studies on this topic. If $c(t)$ is linear, and all the binary variables are fixed to 0 or 1 , (3) reduces to a linear program with non-negative variables and a very nice structure: all constraints are time-precedence constraints, of the form $t_{y}-t_{x} \geq \lambda_{x y}$. This very desirable property will be exploited in the remain of the paper. To this end, we need to manipulate (3) in order to reduce it to a form which is more convenient to our purposes. First, we will show how we can consider a convex piece-wise linear cost function and still maintain the wanted property (Program (8)). Then (8) is simply rewritten in the compact form (9). Finally, we apply a linear transformation to (the coefficient matrix of) (9) so to obtain precisely the dual (BF) of a max-cost flow problem.

\subsection{Cost function and MILP formulation.}

We assume the cost function to be non-negative, piecewise linear and convex, as depicted in Figure 3. Later on in the paper we show that convexity can be relaxed without challenging the validity of the approach.

For every $u \in O$ we let the breakpoints of $c_{u}$ in correspondence to (breakpoint) times $T_{u}^{1}, \ldots, T_{u}^{b(u)}$ - with $b(u)$ being the number of breakpoints. We let $c_{u}^{1}, \ldots, c_{u}^{b(u)}$ be the corresponding non-negative slope increments of the cost function. Namely, for $t_{u} \in\left[T_{u}^{i}, T_{u}^{i+1}\right), i=1, \ldots, b(u)$, the slope will be $c_{u}^{1}+\cdots+c_{u}^{i}\left(\right.$ we let $\left.T_{u}^{b(i)+1}=+\infty\right)$. For every $u \in O$ and times $T_{u}^{1}, \ldots, T_{u}^{b(u)}$, we associate variables $\delta_{u}^{1}, \ldots, \delta_{u}^{b(u)}$ (referred to as delay variables) representing the delay with respect to each breakpoint time. Namely, we have

$$
\delta_{u}^{i}=\max \left(0, t_{u}-T_{u}^{i}\right), \quad i=1, \ldots, b(u)
$$

Then the cost of starting the atomic movement $u$ at time $t_{u}$ is defined as 


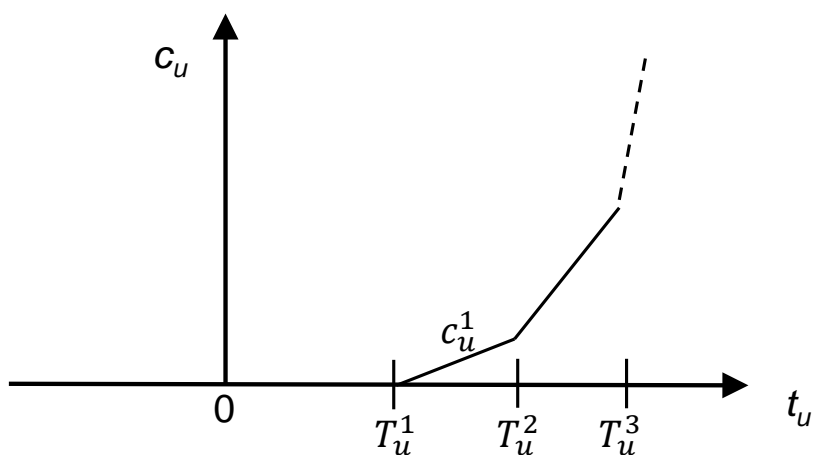

Figure 3: Convex piecewise linear cost function.

$$
c_{u}^{1} \delta_{u}^{1}+\cdots+c_{u}^{b(u)} \delta_{u}^{b(u)}
$$

Since all slope increments are non-negative and we want to minimize the overall cost, we can replace (4) with the following family of constraints in the delay variables:

$$
\begin{gathered}
\delta_{u}^{i} \geq 0, \quad i=1, \ldots, b(u) \\
\delta_{u}^{i} \geq t_{u}-T_{u}^{i} \quad i=1, \ldots, b(u)
\end{gathered}
$$

Problem (3) can now be rewritten as a Mixed Integer Linear Program (MILP):

$\min \quad \sum_{u \in O} \sum_{q=1, \ldots b(u)} c_{u}^{1} \delta_{u}^{1}+c_{u}^{2} \delta_{u}^{2}+\cdots+c_{u}^{b(u)} \delta_{u}^{b(u)}$

s.t.

(i) $\quad t_{v}-t_{u} \geq \lambda_{u v}$

$(u, v) \in A$

(ii) $\quad t_{v}-t_{u} \geq M\left(y_{u v}-1\right)$

$(u, v) \in F$,

(iii) $\quad y_{u v}+y_{w z}=1$,

$\{(u, v),(w, z)\} \in D$,

(iv) $\quad \delta_{u}^{i}-t_{u} \geq-T_{u}^{i}$,

$u \in O, i=1, \ldots, b(u)$,

(v) $\quad \delta_{u}^{i} \geq 0$,

$u \in O, i=1, \ldots, b(u)$,

(vi) $\quad t_{u} \geq 0$

$u \in O$,

$t \in \mathbb{R}^{|O|}, \quad \delta_{u} \in \mathbb{R}^{|b(u)|}, u \in O, \quad y \in\{0,1\}^{|F|}$

The next remarks follow immediately from the definitions.

Remark 2.2 The following facts hold:

1. Only delay variables have non-zero cost coefficients in the objective of (8). 
2. Each delay variable appears in only two constraints of (8), namely one constraint of type (8.iv) and one non-negativity constraint (8.v)

We now simply rewrite (8) in a more compact form. In particular, since all the constraints involving continuous variables are either non-negative constraints, or timeprecedence constraint we can consider a new variable vector $\bar{t}=(t, \delta)$. Renaming all constants accordingly, we can rewrite (8) as

$$
\begin{array}{lll}
\min & \sum_{u \in \bar{V}} \bar{k}_{u} \bar{t}_{u} & \\
\text { s.t. } & \bar{t}_{v}-\bar{t}_{u} \geq \bar{\lambda}_{u v}, & (u, v) \in \bar{A} \\
\text { (i) } & \bar{t}_{v}-\bar{t}_{u} \geq M\left(y_{u v}-1\right) & (u, v) \in F, \\
\text { (ii) } & \{(u, v),(w, z)\} \in D, \\
\text { (iii) } & y_{u v}+y_{w z}=1, & u \in \bar{V}, \\
\text { (iv) } & \bar{t}_{u} \geq 0, & \\
& \bar{t} \in \mathbb{R}^{|\bar{V}|}, \quad y \in\{0,1\}^{|F|} &
\end{array}
$$

where $\bar{V}$ is the set of variable indexes, $\bar{k}_{u} \geq 0$ is the cost of variable $\bar{t}_{u}$ for $u \in \bar{V}$ and $\bar{A}$ is the (extended) family of simple precedence constraints, corresponding to constraints (8.i) and (8.iv). The r.h.s. vector $\bar{\lambda}$ extends vector $\lambda$ to include the (negative of the) breakpoint times appearing in the r.h.s. of $(8 . i v)$. With some abuse of notation we still denote by $D$ the set of (renamed) disjunctive pairs and by $F$ the corresponding set of simple precedence constraints appearing in the disjunctions. Observe that the original delay variables $\delta$ of (8) correspond to a subset $V_{c} \subset V$ of the new set of indices. Again, we have $\bar{k}_{u} \neq 0$ only for variables $\bar{t}_{u}$ with $u \in V_{c}$.

Finally, we further transform the above MILP by introducing a new fictitious movement $o$, the origin. We let $V=\bar{V} \cup\{o\}$, introduce real variables $s_{u}$ for $u \in V$, and let $\bar{t}_{u}=s_{u}-s_{o}$, for $u \in V \backslash\{o\}$. The transformed problem reads as:

$$
\begin{array}{lll}
\min & \sum_{u \in V} k_{u} s_{u} & \\
\text { s.t. } & & (u, v) \in E \\
\text { (i) } & s_{v}-s_{u} \geq l_{u v}, & (u, v) \in F, \\
\text { (ii) } & s_{v}-s_{u} \geq M\left(y_{u v}-1\right) & \{(u, v),(w, z)\} \in D, \\
\text { (iii) } & y_{u v}+y_{w z}=1, & \\
& s \in \mathbb{R}^{|V|}, \quad y \in\{0,1\}^{|F|} &
\end{array}
$$

where $E$ is the resulting family of simple precedence constraints. It is not difficult to see that $k_{u}=\bar{k}_{u}$ for $u \in V \backslash\{o\}$ and $k_{o}=-\left(\sum_{u \in V \backslash\{o\}} k_{u}\right)$. Also, by construction, we have $k_{u} \geq 0$ for all $u \in V \backslash\{o\}$. Observe that the $s$ variables are unconstrained in sign. Indeed, the original set of non-negativity constraints (9.iv) is mapped into the family of simple precedence constraints $s_{u}-s_{o} \geq 0$, for $u \in V$. Finally, we include all simple precedence constraints in the set $E$ and $l$ extends the original vector $\lambda$ accordingly. For 
convenience, we let $l \in \mathbb{R}^{|E \cup F|}$, with $l_{u v}=0$ for $(u, v) \in F$. Note that $V_{c} \subset \bar{V}$, and for $u \in V_{c}$, delay variable $\bar{t}_{u}$ corresponds to (delay) variable $s_{u}$. Next remark derives immediately from Remark 2.2:

Remark 2.3 For $u \in V_{c}$ delay variable $s_{u}$ appears in exactly two constraints of (BF):

1. constraint $s_{u}-s_{w} \geq-T_{a}^{b}$, where $T_{a}^{b}$ is the original breakpoint time associated with $s_{u}$ and $s_{w}$ is the start time of the corresponding atomic movement a.

2. constraint $s_{u}-s_{o} \geq 0$, derived from an original non-negativity constraint.

Finally, observe that through suitable scaling, we may assume that the cost vector $k$ and the length vector $l$ are integral vectors. As a consequence, (BF) is either empty or admits an optimal integer solution ([7]).

\subsection{Disjunctive Graph}

With program $(\mathrm{BF})$ we associate in a standard fashion the directed graph $G=(V, E \cup$ $F$ ), with nodes corresponding to the variables and directed edges associated with simple precedence constraints, including those appearing in a disjunctive constraint. This type of graph was first introduced by Balas in [3], in the context of production scheduling. The vector $l \in \mathbb{R}^{|E \cup F|}$ can be regarded as the lengths of the edges of $G$. The nodes $V_{c} \subset V$ are called delay nodes. The edges in $F$ are called disjunctive edges and the two disjunctive edges associated with an original disjunction are called disjunctive pair (of edges $)^{4}$.

A disjunctive graph associated with two vehicles $A$ and $B$ is depicted in Figure 4. Node 0 is the origin. Nodes 1,2 correspond to the atomic movements of vehicle $A$, whereas 3,4 are those of $B$. There is only one disjunction with corresponding pair of disjunctive edges, $(2,4)$ and $(4,2)$ (dotted in the picture). Nodes 5 and 6 are the delay nodes associated with atomic movement 2 , whereas 7,8 are the delay nodes associated with atomic movement 4 . Edge weights $l_{12}$ and $l_{34}$ are running times whereas $-l_{25},-l_{26}$ $\left(-l_{47},-l_{48}\right)$ are the breakpoints on the time axis of the piecewise linear convex cost function associated with node 2 of train $A$ (node 4 of train $B$ ).

The following facts are simple consequences of the previous definitions and are given without proof.

Property 2.4 The following holds for the disjunctive graph $G$ :

1. For every delay node $u \in V_{c}$, there are exactly two incoming edges and no outgoing edges.

2. There is a directed path from the origin o to all $v \in V$, even when restricted to the set $E$ of non-disjunctive edges.

\footnotetext{
${ }^{4}$ In the literature a disjunctive pair of edges is often denoted as a disjunctive arc
} 


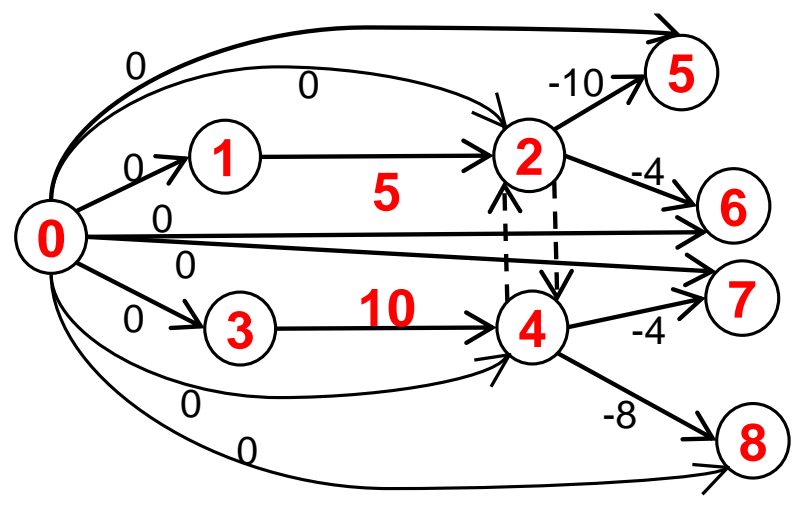

Figure 4: A disjunctive graph associated with two vehicles, $A$ and $B$.

The second property follows from the fact that, for every $v \in V$, there is an edge from $o$ to $v$ of length 0 , deriving from the original non-negativity constraint on the variables. Now, let $\bar{y} \in\{0,1\}^{|F|}$ satisfy constraints (BF.iii). Note that this corresponds to selecting exactly one simple precedence constraint per disjunction, as the other term becomes redundant. So program $(\mathrm{BF})$ reduces to a linear program $(\mathrm{BF}(\bar{y}))$ in the $s$ variables only and with only simple precedence constraints. A natural question is whether $(\mathrm{BF}(\bar{y}))$ admits a feasible solution $\bar{s}, \bar{y}$. A simple answer to this question exploits the properties of the disjunctive graph introduced above.

Note first that vector $\bar{y}$ can be regarded as the incidence vector of a subset $F(\bar{y})$ of disjunctive edges of $G$. Such set $F(\bar{y})$ is called complete selection, because it contains (exactly) one edge for each disjunction in $D$. Let $G(\bar{y})=G(V, E \cup F(\bar{y}))$ be the subgraph of $G$ obtained by dropping all the disjunctive edges in $F \backslash F(\bar{y})$. Observe that the linear program $(\mathrm{BF}(\bar{y}))$ is the dual of a max-cost network flow problem defined on graph $G(\bar{y})$ (we will come back to this in the next section). The following lemma and some other related facts exploited in the following are basic results from network optimization (see, e.g., $[1,7])$.

Lemma 2.5 Let $\bar{y} \in\{0,1\}^{|F|}$ satisfy (BF.iii). Then $(B F(\bar{y}))$ is feasible if and only if the graph $G(\bar{y})$ contains no directed cycles $C$ with strictly positive length $l(C)=$ $\sum_{u v \in C} l_{u v}$.

If $G(\bar{y})$ does not contain a strictly positive length directed cycle, then $G(\bar{y})$ contains a longest directed path $P_{u}^{*}(\bar{y})$ from $o$ to $u$ for every $u \in V$, and we let $L_{u}(\bar{y})=l\left(P_{u}^{*}(\bar{y})\right)$ be its length. Note that $L_{o}(\bar{y})=0$. It is well known (see [7]) that $s_{u}=L_{u}(\bar{y})$, for $u \in V$, is a feasible solution to $(\mathrm{BF}(\bar{y}))$. Also, if $\tilde{s}$ is a feasible solution to $(\mathrm{BF}(\bar{y}))$, then $\bar{s}_{u}=\tilde{s}_{u}-\tilde{s}_{o}$, for $u \in V$ is also feasible for $(\mathrm{BF}(\bar{y}))$ and $\sum_{u \in V} k_{u} \tilde{s}_{u}=\sum_{u \in V} k_{u} \bar{s}_{u}$; also, $\bar{s}_{u} \geq L_{u}(\bar{y})$, for $u \in V{ }^{5}$

\footnotetext{
${ }^{5}$ Indeed, if $P_{u v}$ is a path from $u$ to $v$ in $G(\bar{y})$ and $\bar{s}$ is a feasible solution to $(\mathrm{BF}(\bar{y}))$, we have $\bar{s}_{v} \geq \bar{s}_{u}+l\left(P_{u v}\right)$. Since $\bar{s}_{o}=0$ by construction, then $\bar{s}_{v} \geq l\left(P_{u}^{*}(\bar{y})\right)=L_{u}(\bar{y})$
} 
A feasible complete selection $\bar{F}$ is a complete selection of $F$ such that $G(V, E \cup \bar{F})$ contains no strictly positive directed cycle. We denote by $Y_{f} \subseteq\{0,1\}^{|F|}$ the set of incidence vectors of the feasible complete selections of $F$.

Lemma 2.6 Let $\bar{y} \in Y_{f}$. For $u \in V$, let $L_{u}(\bar{y})$ be the length of a longest path from o to $u$ in $G(\bar{y})$. For $u \in V, s_{u}^{*}=L_{u}(\bar{y})$ is an optimal solution to $(B F(\bar{y}))$.

Proof. First recall that if $(\mathrm{BF}(\bar{y}))$ has an optimal solution, then it has an optimal solution $\tilde{s}$ with $\tilde{s}_{o}=0([7])$. The cost of such solution will be $c(\tilde{s})=\sum_{u \in V_{c}} k_{u} \tilde{s}_{u}$ since $k_{u}=0$ for all $u \notin V_{c} \cup\{o\}$ and $\tilde{s}_{o}=0$. Now, it is well known that $s^{*}=L(\bar{y})$ is feasible for $(\mathrm{BF}(\bar{y}))$ and we have $s_{o}^{*}=0$. Moreover, let $\bar{s}$ be any feasible for $(\mathrm{BF}(\bar{y}))$ with $\bar{s}_{o}=0$ : then $\bar{s} \geq s^{*}$. The Lemma follows from the fact that for $u \in V_{c} . k_{u} \geq 0$ and thus $c(\bar{s})=\sum_{u \in V_{c}} k_{u} \bar{s}_{u} \geq \sum_{u \in V_{c}} k_{u} s_{u}^{*}=c\left(s^{*}\right)$.

The cost $c\left(s^{*}\right)$ of such optimal solution is thus $\sum_{u \in V} k_{u} L_{u}(\bar{y})$. Also, since $L_{u}(\bar{y}) \geq 0$ for all $u \in V$ (due to the 0-length edge from $o$ to $u$ ), then any feasible solution has non-negative cost. Now, from basic network optimization theory we know that $L_{u}(\bar{y})$ corresponds to the length of the unique path from $o$ to $u$ in a certain directed spanning tree of $G(\bar{y})$ rooted at o called longest-path tree. Summarizing:

Property 2.7 For $y \in Y_{f}$, the optimal schedule for $B F(\bar{y})$ is in correspondence to a longest-path tree (rooted in o) of $G(\bar{y})$.

Such spanning trees are branchings (see [29]) since the in-degree of each node is 1 , except for the root $o$ (with in-degree 0 ).

Note that the proof of the above Lemma relies on the crucial property that every feasible solution $s$ to $(\mathrm{BF}(\bar{y}))$ such that $s_{o}=0$ is such that each component $s_{u}$ is not smaller than $L_{u}(\bar{y})$. This allows for an immediate generalization to any non-decreasing non-negative cost function:

Corollary 2.8 Let $c: \mathbb{R}^{|V|} \rightarrow \mathbb{R}$ be a non-decreasing non-negative function, let $\bar{y} \in Y_{f}$ and consider a problem $Q(\bar{y})$ obtained from $(B F(\bar{y}))$ by replacing the original objective function with $c(s)$ and including the constraint $s_{o}=0$. Let $T(\bar{y})$ be a longest path tree in $G(\bar{y})$. Then $s_{u}^{*}=L_{u}(\bar{y})$ is an optimal solution for $Q(\bar{y})$, where $L_{u}(\bar{y})$ is the length of the unique path from o to $u$ in $T(\bar{y})$.

So, let $T=T(\bar{y})$ be a longest-path tree (from now on all our longest path trees will be directed spanning trees rooted at $o$ ) of $G(\bar{y})$. We define the $\operatorname{cost} c(T)$ of $T$ as the cost of the solution associated with $\mathrm{BF}(\bar{y})$, namely $c(T)=\sum_{u \in V_{c}} k_{u} L_{u}(\bar{y})$. Since $L_{u}(\bar{y})$ is the length $L_{u}(T)$ of the unique path $P_{u}(T)$ from $o$ to $u$ in $T$, we can rewrite

$$
c(T)=\sum_{u \in V_{c}} k_{u} L_{u}(T)=\sum_{u \in V_{c}} c\left(P_{u}(T)\right)
$$


where we define $c\left(P_{u}(T)\right)=k_{u} L_{u}(T)$ as the cost of path $P_{u}(T)$.

Finally, observe that, because a delay node $u \in V_{c}$ has in-degree 2 and out-degree 0 (see Property 2.4), then the following holds.

Property 2.9 Let $T$ be a longest path tree of $G(\bar{y})$ rooted at o and let $u \in V_{c}$ be a delay node. Then $u$ is a leaf of $T$.

In Figure 5 we give an example. The solid edges are the edges of a longest-path tree $T$ of $G(\bar{y})$ for $\bar{y}_{24}=1, \bar{y}_{42}=0$. Delay nodes are (the only) leaves of $T$. The associated solution in the delay nodes is $s_{6}=1, s_{7}=0, s_{8}=7, s_{9}=3$. Assuming $k_{6}=3, k_{7}=4, k_{8}=5, k_{9}=3$, we have $c(T)=47$.

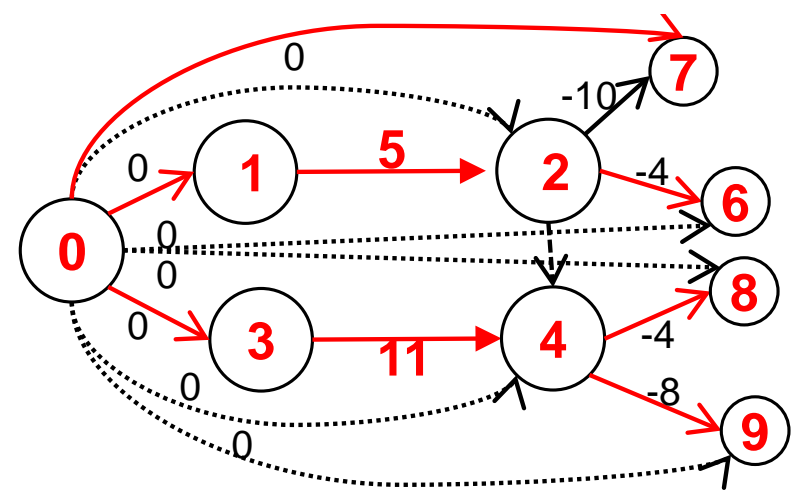

Figure 5: Longest-path tree $T$ of $G(\bar{y})$.

\section{Benders' decomposition and reformulation}

We now rewrite program (BF) by applying the classical Benders' reformulation ([23]). For any vector $y \in\{0,1\}^{|F|}$ satisfying (BF.iii), program (BF) reduces to the dual of the following max-cost flow problem:

$$
\begin{array}{lll}
\max & \sum_{(u, v) \in E} l_{u v} x_{u v}+\left(\sum_{(u, v) \in F} M\left(y_{u v}-1\right) x_{u v}\right. & \\
\text { s.t. } & \sum_{u v \in \delta^{-}(v)} x_{u v}-\sum_{v u \in \delta^{+}(v)} x_{v u}=k_{v}, & v \in V \\
\text { (i) } & \text { uv } \in E \cup F \\
\text { (ii) } & x_{u v} \geq 0, &
\end{array}
$$

where $\delta^{-}(v)$ and $\delta^{+}(v)$ are the incoming and outgoing stars of $v \in V$ in graph $G=$ $(V, E \cup F)$. We let $P(G)$ be the polyhedron of the feasible points of $(M F(y))$.

When $y$ is the incidence vector of a complete selection and $G(y)$ does not contain strictly positive length cycles (i.e. $y \in Y_{f}$ ), the following holds: 
Lemma 3.1 Let $y \in Y_{f}$, and let $H$ be a longest path tree of $G(y)$ of $\operatorname{cost} c(H)$. Then

1. $M F(y)$ has an optimal feasible solution $x(y)=p^{*}$, where $p^{*}$ is an extreme point of $P(G)$ and $p_{e}^{*}=0$ for all $e \notin H$.

2. The value of the optimal solution is $v\left(p^{*}\right)=\sum_{(u, v) \in H \cap E} l_{u v} p_{u v}^{*}$

3. We have $v\left(p^{*}\right)=c(H)$

Proof. 1.) and 3.) derive directly from classical duality results (see, e.g., [7]). As for 2.), let $y$ be the incidence vector of $F(y) \subseteq F$ and so by construction $H \subseteq E \cup F(y)$. By 1.) we have that, for $e \in F, p_{e}^{*}>0$ implies $e \in F(y)$, and thus $y_{e}=1$. So, for $e \in F$ we have $p_{e}^{*}\left(1-y_{e}\right)=0$ and $\sum_{(u, v) \in F} M\left(y_{u v}-1\right) p_{u v}^{*}=0$.

Let $K$ and $J$ be the set of extreme points $p^{k}(k \in K)$ and extreme rays $r^{j}(j \in J)$ of $P(G)$, respectively. Observe that since they are feasible flows, all extreme points and rays are non-negative vectors. Also, since we assume the r.h.s. $k_{v}$ to be integer for all $v \in V$, then $P(G)$ is an integral polyhedron and all its vertices are integral points ([7]). Benders' reformulation of $(\mathrm{BF})$ writes as

$\min \quad \eta$

s.t.

(i) $\quad y_{u v}+y_{w z}=1, \quad\{(u, v),(w, z)\} \in D$,

(ii) $\quad \sum_{e \in E} l_{e} r_{e}^{j}+\sum_{e \in F} M\left(y_{e}-1\right) r_{e}^{j} \leq 0, \quad j \in J$,

$$
\begin{aligned}
& \sum_{e \in E} l_{e} p_{e}^{i}+\sum_{e \in F} M\left(y_{e}-1\right) p_{e}^{i} \leq \eta, \quad i \in K, \\
& y \in\{0,1\}^{|F|}, \eta \in \mathbb{R}_{+}
\end{aligned}
$$

Constraints (11.ii) are called feasibility cuts, whereas constraints (11.iii) are the optimality cuts. We remark that, since any feasible solution to (BF) has non-negative cost, we have that also $\eta$ is non-negative in every feasible solution to (11), and we explicitly stipulate this.

Note that the reformulation only contains the original binary variables $y$ (one for each disjunctive edge), plus one continuous variable $\eta$ representing the cost of the solution. However, the notorious big- $M$ coefficients are still present in the constraints. In the remaining of this section we exploit classical results from polyhedral combinatorics and network optimization to finally get rid of such coefficients. In particular, the following definitions and results may be found in [4]. Let $S=\left\{z \in\{0,1\}^{|K|}: \sum_{i \in K} a_{i} z_{i} \leq b\right\}$ be the feasible solution set to the knapsack constraint $\sum_{i \in K} a_{i} z_{i} \leq b$, where $a_{i} \in Z_{+}$. A cover (of the knapsack constraint) is a subset $Q \subseteq K$ such that $\sum_{i \in Q} a_{i}>b$. The cover inequality associated with a cover $Q$ is $\sum_{i \in Q} z_{i} \leq|Q|-1$ and is satisfied by every $z \in S$. A cover $Q$ is minimal if no proper subset of $Q$ is a cover. The cover inequality associated with a minimal cover is facet defining for $\operatorname{conv}(S)$. A 
stronger formulation for $S$ can be obtained by replacing the original knapsack constraint with the cover constraints associated with the set $\mathcal{Q}$ of its minimal covers, namely $S=\left\{z \in\{0,1\}^{|K|}: \sum_{i \in Q} z_{i} \leq|Q|-1, \quad Q \in \mathcal{Q}\right\}$.

Lemma 3.2 The set of constraints (11.ii) can be replaced by the following set of constraints:

$$
\sum_{e \in F \cap C} y_{e} \leq|F \cap C|-1 \quad C \in \mathcal{C}
$$

where $\mathcal{C}$ is the set of strictly positive length directed cycles of $G$.

Proof. It is well known (see e.g. [7]) that each extreme ray $r^{C} \in P(G)$ is the incidence vector of a directed cycle $C$ in $G$. Consider the constraint (11.ii) associated with $C$. Now, if $l(C)=\sum_{e \in C} l_{e}=\sum_{e \in E \cup F} l_{e} r_{e}^{C}=\sum_{e \in E} l_{e} r_{e}^{C} \leq 0$, then (11.ii) is trivially satisfied, because $\sum_{e \in F}\left(y_{e}-1\right) \leq 0$ for any $y \in\{0,1\}^{|F|}$. So, we can restrict (11.ii) to the set $\mathcal{C}$ of strictly positive length directed cycles of $G$. Now, let $C \in \mathcal{C}$ and let $\sum_{e \in E \cap C} l_{e}+\sum_{e \in F \cap C} M\left(y_{e}-1\right) \leq 0$ be the knapsack constraint (11.ii) associated with (the incidence vector of) $C$. Then it is not difficult to see that the knapsack has a unique minimal cover $F \cap C$.

Incidentally, constraint (12) can also be interpreted as a "combinatorial Benders' cut" (see [11]). It has a direct interpretation: for each strictly positive directed cycle $C$ of $G$ and each selection vector $y$, at least one of the disjunctive edges contained in $C$ cannot be selected by $y$.

Next, we focus on constraints (11.iii) associated with the extreme points of $P(G)$. Each extreme point of $P(G)$ is in one-to-one correspondence with the set $\mathcal{T}$ of spanning trees of $G$ corresponding to the feasible basis of $(M F(y))$, for $y \in Y_{f}$ (see [7]). In particular, by Lemma 3.1 if $p^{H} \in P(G)$ is the extreme point corresponding to spanning tree $H \in \mathcal{T}$, we have that $p_{e}^{H}=0$ for all $e \notin H$. In order to highlight this mapping, we rewrite the set (11.iii) as:

$$
\sum_{e \in E \cap H} l_{e} p_{e}^{H}+\sum_{e \in F \cap H} M\left(y_{e}-1\right) p_{e}^{H} \leq \eta, \quad H \in \mathcal{T}
$$

We will now show that the set $\mathcal{T}$ can be restricted to the family of longest path trees of $G(y)$, for $y \in Y_{f}$. Observe that if $y \in\{0,1\}^{|F|}$ satisfies (11.i) and (11.ii), then $y$ is the incidence vector of a complete selection and $G(y)$ does not contain strictly positive length cycles, so $y \in Y_{f}$. In other words, for $y \notin Y_{f}$, constraints (11.iii) become redundant. When $y \in Y_{f}$ we have the following:

Lemma 3.3 The set of constraints (11.iii) is dominated by the set of valid inequalities

$$
\sum_{e \in E \cap H} l_{e} p_{e}^{H}+\sum_{e \in F \cap H} M\left(y_{e}-1\right) p_{e}^{H} \leq \eta, \quad H \in \mathcal{T}^{*}
$$

where $\mathcal{T}^{*}=\left\{H^{*}(y): y \in Y_{f}, H^{*}(y)\right.$ any longest path tree in $\left.G(y),\right\}$. 
Proof. We assume $Y_{f} \neq \emptyset$ (otherwise problem (11) is trivial or infeasible). Let $\bar{y} \in Y_{f}$ and let $\mathcal{T}(\bar{y})$ be the set of spanning trees associated with the feasible basis of $(M F(y))$. For $H \in \mathcal{T}(\bar{y})$, the 1.h.s. of constraint (13) is dominated by

$$
\max _{H \in \mathcal{T}(\bar{y})}\left(\sum_{e \in E \cap H} l_{e} p_{e}^{H}+\sum_{e \in F \cap H} M\left(\bar{y}_{e}-1\right) p_{e}^{H}\right) \leq \eta,
$$

By Lemma 3.1 the maximum above is obtained for $H^{*}$ longest path tree of $G(\bar{y})$.

By statement 3.) of Lemma 3.1, for $H \in \mathcal{T}^{*}$, each constraint (14) can also be written as

$$
c(H)+\sum_{e \in F \cap H} M\left(y_{e}-1\right) p_{e}^{H} \leq \eta
$$

It is not difficult to see that, for $y \in\{0,1\}^{|F|}$, the above constraint is satisfied if and only if $y$ satisfies the following inequality:

$$
c(H)\left(\sum_{e \in F \cap H} y_{e}-|F \cap H|+1\right) \leq \eta,
$$

In fact, one can show a stronger result still. Let $H \in \mathcal{T}^{*}$. For $e \in F \cap H$, let $M_{e}=p_{e}^{H} \cdot M$. Note that $p_{e}^{H}>0$ for some $e \in F \cap H$, implies $M_{e} \geq M$, because $p^{H}$ is integral. Let $N=\left\{e \in F \cap H: M_{e}>0\right\}$.

Lemma 3.4 Let $Y^{H}=\left\{(y, \eta) \in\{0,1\}^{|F \cap H|} \times \mathbb{R}_{+}^{1}:(y, \eta)\right.$ satisfies $\left.(15)\right\}$. Then the constraint

$$
c(H)\left(\sum_{e \in N} y_{e}-|N|+1\right) \leq \eta,
$$

is facet defining for $\operatorname{conv}\left(Y^{H}\right)$.

The proof of Lemma 3.4 can be found in the appendix.

So, when $N=F \cap H$, we have that (16) is facet defining for $\operatorname{conv}\left(Y^{H}\right)$; otherwise it is the trivial lifting of a facet defining inequality. Incidentally, note that one can easily prove that, for $e \in F \cap H$, we have $p_{e}^{H}>0$ (i.e. $e \in N$ ) if and only if $e$ is on the unique path in $H$ from $o$ to some delay node $v \in V_{c}$, that is to a node $v$ such that $k_{v}>0$. The variables corresponding to these disjunctive edges are the only ones appearing in the constraint (17). We somehow exploit this observation in our main result, stated in Theorem 3.5. To this end, recall that for $H \in \mathcal{T}^{*}$, its cost $c(H)$ can be computed as $\sum_{u \in V_{c}} c\left(P_{u}(H)\right)$ where $V_{c}$ is a set of leaves of $H$ and $c\left(P_{u}(H)\right)=k_{u} L_{u}(H)$ is the cost of the unique path $P_{u}(H)$ from $o$ to $u$ in $H$. The following theorem holds: 
Theorem 3.5 Let $H \in \mathcal{T}^{*}$ and let $\mu_{u} \in \mathbb{R}$, for $u \in V_{c}$. Then constraint (16) is dominated ${ }^{6}$ by the following system of valid inequalities:

$$
\begin{aligned}
& \eta=\sum_{u \in V_{c}} \mu_{u}, \\
& c\left(P_{u}(H)\right)\left(\sum_{e \in F \cap P_{u}(H)} y_{e}-\left|F \cap P_{u}(H)\right|+1\right) \leq \mu_{u}, \quad u \in V_{c} .
\end{aligned}
$$

Before proving our main theorem, we prove an intermediate lemma. Let $L_{1}$ and $L_{2}$ be a partition of $V_{c}$ and let $H_{1}\left(H_{2}\right)$ be the subtree of $H$ containing only the edges in the paths $P_{u}(H)$, from the origin $o$ to every node $u \in L_{1}\left(u \in L_{2}\right)$ (see Figure 6). Let $c\left(H_{1}\right)=\sum_{u \in L_{1}} c\left(P_{u}\right)$ be the cost of $H_{1}$ and $c\left(H_{2}\right)=\sum_{u \in L_{2}} c\left(P_{u}\right)$ be the cost of $H_{2}$. Clearly $c(H)=c\left(H_{1}\right)+c\left(H_{2}\right)$. Note that $H_{1}$ and $H_{2}$ may intersect. Finally, let $F_{0}=H \cap F, F_{1}=H_{1} \cap F$ and $F_{2}=H_{2} \cap F$ be the set of disjunctive edges in $H, H_{1}$ and $H_{2}$, respectively. Note that

$$
F_{0}=F_{1} \cup F_{2}=\left(F_{1} \backslash F_{2}\right) \cup\left(F_{2} \backslash F_{1}\right) \cup\left(F_{1} \cap F_{2}\right)
$$
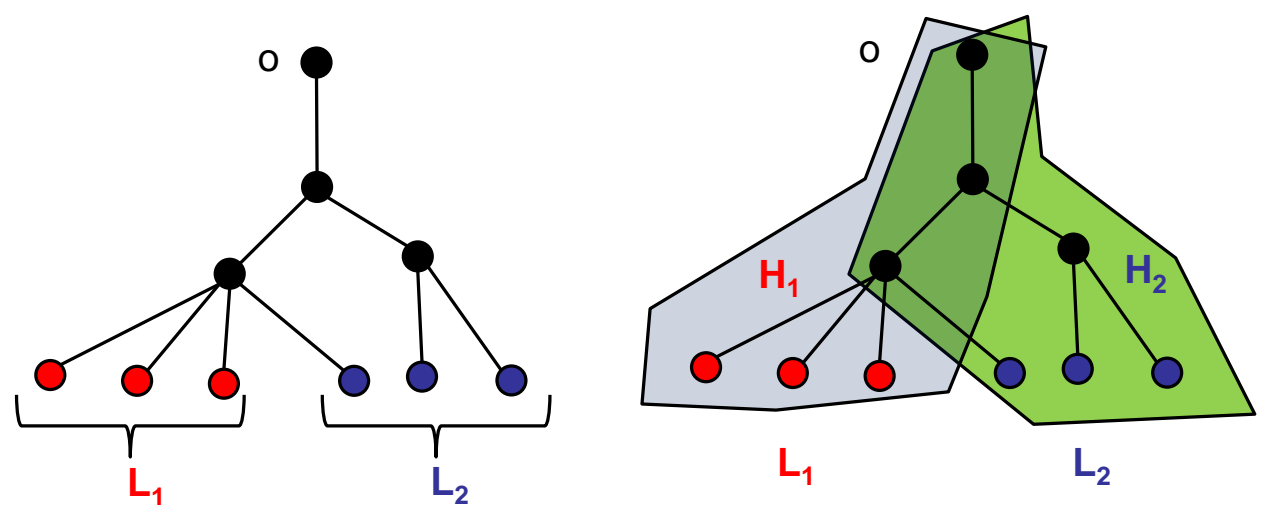

Figure 6: The bipartition of the leaves of $H$ and the corresponding trees $H_{1}$ and $H_{2}$

Lemma 3.6 Constraint (16) is dominated by the following system of valid inequalities:

$$
\begin{array}{lll}
\text { (i) } & \eta=\mu_{1}+\mu_{2}, & , \\
\text { (ii) } & c\left(H_{1}\right)\left(\sum_{e \in F_{1}} y_{e}-\left|F_{1}\right|+1\right) \leq \mu_{1}, & i \in L_{1}, \\
\text { (iii) } & c\left(H_{2}\right)\left(\sum_{e \in F_{2}} y_{e}-\left|F_{2}\right|+1\right) \leq \mu_{2}, & i \in L_{2},
\end{array}
$$

Proof. The validity of (20) is trivial. Next, note that system (20) dominates the surrogate constraint

\footnotetext{
${ }^{6} \mathrm{By}$ "dominated" we mean here that if the real vector $(\bar{y}, \bar{\eta}, \bar{\mu})$ is feasible for (18) then its projection $(\bar{y}, \bar{\eta})$ is feasible for $(16)$
} 


$$
c\left(H_{1}\right)\left(\sum_{e \in F_{1}} y_{e}-\left|F_{1}\right|+1\right)+c\left(H_{2}\right)\left(\sum_{e \in F_{2}} y_{e}-\left|F_{2}\right|+1\right) \leq \mu_{1}+\mu_{2}=\eta .
$$

We show now that the above constraint dominates (16) by proving that, for any $y \in$ $[0,1]^{F}$, we have:

$c\left(H_{1}\right)\left(\sum_{e \in F_{1}} y_{e}-\left|F_{1}\right|+1\right)+c\left(H_{2}\right)\left(\sum_{e \in F_{2}} y_{e}-\left|F_{2}\right|+1\right) \geq\left(c\left(H_{1}\right)+c\left(H_{2}\right)\right)\left(\sum_{e \in F_{0}} y_{e}-\left|F_{0}\right|+1\right)$

or, after simplification:

$$
c\left(H_{1}\right)\left(\sum_{e \in F_{1}} y_{e}-\left|F_{1}\right|\right)+c\left(H_{2}\right)\left(\sum_{e \in F_{2}} y_{e}-\left|F_{2}\right|\right) \geq\left(c\left(H_{1}\right)+c\left(H_{2}\right)\right)\left(\sum_{e \in F_{0}} y_{e}-\left|F_{0}\right|\right)
$$

Observe that $F_{1}$ can be partitioned in $F_{1} \backslash F_{2}$ and $F_{1} \cap F_{2}$; similarly $F_{2}$ can be partitioned in $F_{2} \backslash F_{1}$ and $F_{1} \cap F_{2}$ : then the left-hand side of (21) can be written as:

$$
c\left(H_{1}\right)\left(\sum_{e \in F_{1} \backslash F_{2}} y_{e}-\left|F_{1} \backslash F_{2}\right|\right)+c\left(H_{2}\right)\left(\sum_{e \in F_{2} \backslash F_{1}} y_{e}-\left|F_{2} \backslash F_{1}\right|\right)+\left(c\left(H_{1}\right)+c\left(H_{2}\right)\right)\left(\sum_{e \in F_{1} \cap F_{2}} y_{e}-\left|F_{2} \cap F_{1}\right|\right)
$$

While, by using (19), the right-hand side of (21) writes as:

$$
\left(c\left(H_{1}\right)+c\left(H_{2}\right)\right)\left(\sum_{e \in F_{1} \backslash F_{2}} y_{e}-\left|F_{1} \backslash F_{2}\right|+\sum_{e \in F_{2} \backslash F_{1}} y_{e}-\left|F_{2} \backslash F_{1}\right|+\sum_{e \in F_{1} \cap F_{2}} y_{e}-\left|F_{2} \cap F_{1}\right|\right)
$$

Substituting (22) and (23) in (21) and simplifying, we obtain:

$$
0 \geq c\left(H_{2}\right)\left(\sum_{e \in F_{1} \backslash F_{2}} y_{e}-\left|F_{1} \backslash F_{2}\right|\right)+c\left(H_{1}\right)\left(\sum_{e \in F_{2} \backslash F_{1}} y_{e}-\left|F_{2} \backslash F_{1}\right|\right)
$$

which is trivially satisfied for any $y \in[0,1]^{F}$ since $c\left(H_{1}\right), c\left(H_{2}\right) \geq 0$

In order to prove Theorem 3.5, we can use induction: if both $L_{1}$ and $L_{2}$ are singletons, we are done; otherwise we can (recursively) apply Lemma 3.6 to $H_{1}$ and $H_{2}$ separately.

We can finally rewrite problem (11) as follows 
$\min \quad \sum_{u \in V_{c}} \mu_{u}$

s.t.

(i) $\quad y_{u v}+y_{w z}=1$,

$\{(u, v),(w, z)\} \in D$,

(ii) $\quad \sum_{e \in C \cap F} y_{e} \leq|C \cap F|-1$,

$C \in \mathcal{C}$,

(iii)

$$
\begin{aligned}
& c\left(P_{u}(H)\right)\left(\sum_{e \in F \cap P_{u}(H)} y_{e}-\left|F \cap P_{u}(H)\right|+1\right) \leq \mu_{u}, \quad u \in V_{c}, H \in \mathcal{T}^{*} \\
& y \in\{0,1\}^{|F|}, \eta \in \mathbb{R}_{+}
\end{aligned}
$$

Extension to separable, non-decreasing non-negative cost functions. Now, consider non decreasing non-negative functions $c_{u}: \mathbb{R} \rightarrow \mathbb{R}$, for $u \in V_{c}$ and, for $s \in \mathbb{R}^{|V|}$, let $c(s)=\sum_{u \in V_{c}} c_{u}\left(s_{u}\right)$. In other words, we assume $c$ to be separable, and the non-zero components of $c$ correspond to the nodes in $V_{c}$. As in corollary 2.8, consider problem $Q(\bar{y})$ obtained from $(\mathrm{BF}(\bar{y}))$ by replacing the original objective function with $c(s)$. Then it is not difficult to see that problem $Q(\bar{y})$ can be reformulated as (Bend), where $c\left(P_{u}(H)\right)$ is defined as $c_{u}\left(L_{u}(H)\right)$ and $L_{u}(H)$ is the length of $P_{u}(H)$.

A final observation, even if $c$ is a non-separable (non-decreasing non-negative) function we can still reformulate the original problem by using the weaker inequalities (16) instead of (Bend.iii) (and considering the original objective $\min \eta$ ).

\section{Solution Algorithm}

To solve MILP (Bend) we use classical delayed row and column generation [2,13]. Recall that, given an optimal solution $y^{*}$ to (Bend), the associated optimal schedule $s^{*}=s\left(y^{*}\right)$ can be computed by a longest path tree computation in graph $G\left(y^{*}\right)=\left(V, E \cup F\left(y^{*}\right)\right)$, where $F\left(y^{*}\right)$ is the set of disjunctive edges "selected" by $y^{*}$.

Our algorithm solves a sequence of subproblems $\left(\right.$ Bend $\left.^{1}\right),\left(\right.$ Bend $\left.^{2}\right), \ldots$, until an optimal solution is found or the problem is (proven) infeasible. Problem $\left(\mathrm{Bend}^{k}\right)$ is associated with the triple $\left(D^{k}, \mathcal{C}^{k}, \mathcal{V} \mathcal{T}^{k}\right)$ representing the current subset of (indices of) constraints (Bend.i), (Bend.ii) and (Bend.iii), respectively (with $\mathcal{V} \mathcal{T}^{k} \subseteq V_{c} \times \mathcal{T}^{*}$ ). Also, we let $y^{k}$ be the optimal solution to $\left(\operatorname{Bend}^{k}\right)$, with $y_{u v}=y_{w z}=0$ for all $\{(u, v),(w, z)\} \in$ $D \backslash D^{k}$. So, if $D^{k} \neq D$, the selection $F\left(y^{k}\right)$ is not a complete selection, since some of the original disjunctive pairs are not taken into account as the corresponding pairs of variables are fixed to 0 . We denote by $F^{k}$ the set of disjunctive edges associated with the disjunctions of $D^{k}$ and by $G\left(y^{k}\right)=G\left(V, E \cup F^{k}\right)$. Also, we let $s^{k}=s\left(y^{k}\right)$ be the associated optimal schedule, calculated by solving $\left(\mathrm{BF}\left(y^{k}\right)\right)$, namely problem $\left.(\mathrm{BF})(y)\right)$ restricted to the constraints in $E \cup F^{k}$ and to the disjunctions of $D^{k}$.

The algorithm proceeds by growing sets $D^{k}, \mathcal{C}^{k}, \mathcal{V} \mathcal{T}^{k}$. Namely we have $\emptyset=D^{0} \subseteq$ $D^{1} \subseteq \ldots, \emptyset=\mathcal{C}^{0} \subseteq \mathcal{C}^{1} \subseteq \ldots$ and $\emptyset=\mathcal{V} \mathcal{T}^{0} \subseteq \mathcal{V} \mathcal{T}^{1} \subseteq \ldots$ 
At each iteration we first check if $y^{k}$ violates a cycle inequality $C$. If so, we add $C$ to the set of cycle inequalities and iterate. If not, we compute a longest path tree $T^{k}$ in $G\left(y^{k}\right)$ and check whether some path inequalities associated with $T^{k}$ are violated. If so, we add them to the set of path inequalities and iterate. Otherwise we perform a macro-iteration, namely we check if solution $s^{k}$ associated with $T^{k}$ violates any of the disjunctions in the set $D \backslash D^{k}$. If not, we are done: we let $s^{*}=s^{k}$, we (trivially) extend $y^{k}$ to a feasible complete selection $y^{*}$ respecting $s^{*}$ and we have that $\left(s^{*}, y^{*}\right)$ is an optimal solution to our original problem. Otherwise, we add the violated disjunction $\{(u, v),(w, z)\}$ to the current set $D^{k}$ and iterate.

\section{Paths\&Cycles Algorithm (PC)}

0 . Set $k=0 ;$ Set $D^{1}=\emptyset, \mathcal{C}^{1}=\emptyset, \mathcal{V} \mathcal{T}^{1}=\emptyset$.

\section{$\underline{\text { Micro Iterations }}$}

1. $k=k+1$. Solve $\left(\right.$ Bend $\left.^{k}\right)$. If $\left(\right.$ Bend $\left.^{k}\right)$ infeasible, (Bend) is infeasible and STOP.

2. Let $y^{k}$ be the optimal solution to $\left(\operatorname{Bend}^{k}\right)$.

3. If $y^{k}$ violates cycle inequality $C$ :

Let $\mathcal{C}^{k+1}=\mathcal{C}^{k} \cup\{C\}, D^{k+1}=D^{k}, \mathcal{V} \mathcal{T}^{k+1}=\mathcal{V} \mathcal{T}^{k}$. GoTo 1 .

4. Solve $\left(\mathrm{BF}\left(y^{k}\right)\right)$. Let $T^{k} \in \mathcal{T}^{*}$ be the longest path tree of $G\left(y^{k}\right)$ and $s^{k}$ be the associated schedule.

5 If $y^{k}$ violates path inequalities $V T^{k}=\left\{\left(v, T^{k}\right): v \in V_{c}^{k} \subseteq V_{c}\right\}$ :

Let $\mathcal{V} \mathcal{T}^{k+1}=\mathcal{V} \mathcal{T}^{k} \cup V T^{k}, D^{k+1}=D^{k}, \mathcal{C}^{k+1}=\mathcal{C}^{k}$. GoTo 1 .

Macro Iterations

6. If $s^{k}$ violates disjunction $d$, let $D^{k+1}=D^{k} \cup\{d\} . \mathcal{C}^{k+1}=\mathcal{C}^{k}, \mathcal{V} \mathcal{T}^{k+1}=\mathcal{V} \mathcal{T}^{k}$.

\section{GoTo 1}

7. STOP: extend $y^{k}$ to a complete selection $y^{*}$, and $\left(y^{*}, s^{*}=s^{k}\right)$ is an optimal solution for $(\mathrm{BF})$.

The solution of the 0,1 program $\left(\right.$ Bend $\left.^{k}\right)$ at Step 1 can be obtained by invoking any MILP solver (see Section 5). Problem $\left(\mathrm{BF}\left(y^{k}\right)\right)$ amounts to a longest path tree computation in $G\left(y^{k}\right)$, which in turn can be obtained in $O\left(\left|E \cup F\left(y^{k}\right)\right|\right)$ for acyclic graphs (which is the case for the real-life instances presented in Section 5, and more generally for many relevant traffic management problems). Note that, in each macro iteration $k$, only a subset of the $y$ variables can be different from 0 , namely variables $y_{e}$ for $e \in F^{k}$. One can then neglect all other variables $y_{e}$ for $e \in F \backslash F^{k}$. So, at iteration $k$ only variables $y_{e}$ for $e \in F^{k}$ are actually included in the current MILP (Bend ${ }^{k}$ ) (delayed column generation). 


\section{Computational Results}

In this section we present computational results for the $\mathrm{PC}$ algorithm. We test the algorithm on real-life instances from train rescheduling, an operational problem that arises in managing real-time railway traffic. This is in fact the application which originally motivated this new study: the necessity to improve on a successful but big- $M$ based approach (referred to as $B M$ ) presented in [18] (which extends and completes the formulation introduced in [17]). In $B M$, the big- $M$ formulation (3) is solved repeatedly following the macroscopic/microscopic decomposition approach described in [30]. In this paper, we compare $B M$ with the new $P C$ algorithm. $B M$ is also based on delayed row generation as the $P C$ algorithm (see Section 4); however, at Step 1, formulation (Bend) is replaced by the initial big- $M$ formulation (3) introduced in Section 2. Consequently, Steps 2, 3, 4, and 5 of the PC Algorithm are skipped, while 6 and 7 are executed. For more detail regarding $B M$ we refer to ([18]).

The instances are provided by a train TMS (traffic management system) operative on a railway line in Norway, in the Stavanger region (see Section 5.1). In the rest of the section, we first present information about the test instances and the implementation details, then present comparisons between $B M$ and $P C$ for different types of nondecreasing non-negative objective functions that typically arise in this kind of traffic management problems.

\subsection{Test instances}

First, we give some context regarding the instances used in this paper for our tests. All instances refer to real-life trains running on a railway line at a certain moment in time. More precisely, a snapshot of the state of the system (train position, network status) is taken each time an event occurs on the line (train reaches signaling point, delays are registered etc.). The instances are provided by an optimization-based train rescheduling system implemented in Norway in February 2014. The system was developed at SINTEF and funded by Norway's infrastructure manager (Jernbaneverket), train operating companies (NSB, Flytoget, CargoNet) and Research Council (Forskningsrådet). The system was implemented on the main line of the Stavanger region (the Joerbane) on the west coast of Norway (also including the railway between Egersund and Moi). The Jærbane $(123 \mathrm{~km}, 16$ stations) is one of the few lines in Norway with both singleand double-tracks and is the most trafficked line in Norway outside of the Oslo area.

As mentioned in the introduction, the problem can be decomposed into "station problems" and a "line problem", and in this paper we only focus on the latter (in [18] we show how the two problems can be re-coupled exactly by means of Benders' logic decomposition). The line problem can be viewed as a problem where each station is collapsed into a single, capacitated node (and thus we call it a macroscopic representation of the railway). So, in the line problem the railway is essentially reduced to a sequence of nodes representing the original stations, connected by single or double-track sections. In single track sections, trains can only meet or pass in stations. In double 
track sections, each track is devoted to one of the two directions, so meets can happen anywhere, whereas passes can only occur in stations. In other words, the line problem consists in finding a schedule for the trains running during the planning horizon such that meets and passes occur only in feasible regions of the network and some cost associated with the delays is minimized. In our instances, trains traveling along the entire line have approximately 50 (macroscopic) atomic movements. ${ }^{7}$ Even though neglected in this paper, it is worth mentioning that each station problem is a feasibility problem that, besides scheduling, also involves routing trains. Indeed, in many stations trains can choose among several routes and platforms. In [18] we show how this problem can be modelled and solved. However, we remark here that, in our instances, the bottleneck of the overall solution process is the solution to the line problem, requiring significantly higher computational effort.

\section{$5.2 \quad$ Implementation details}

Tests were run on a Dell laptop with an Intel (R) Core (TM) i7-5600U CPU @2.60 GHz 16GB RAM. IBM Cplex 12.532 bit was used for all experiments, with the exception of some of the instances in Tables 3 and 6 for which the 64-bit version was used due to memory issues when solving the MILPs (more info is given in describing the tables).

\subsection{Convex non-decreasing (non-negative) piecewise linear cost functions}

Convex non-decreasing (non-negative) objective functions are commonly adopted for job-shop scheduling problems in traffic management. Such objectives can typically be expressed or approximated with piece-wise linear functions (see Figure 3). Broadly speaking, if the system is in a state that requires recovery, e.g. train $i$ is delayed, a convex objective function enforces that the delay will be somehow spread out on the trains encountered by $i$ (assuming, for instance, these are not themselves already delayed). Convex functions are chosen to model recovery decisions for a combination of reasons. First of all, they model the behaviour/recovery strategies of planners (or controllers, dispatchers etc.) reasonably well, particularly in situations that are not unusually critical (like when one or more vehicles are extremely delayed or there are large scale disruptions). Secondly, they are deemed preferable on the modeling side due to their computational tractability. Modeling a convex objective function for $B M$ only requires adding an extra continuous variable for each interval (or "piece") of the objective and some linear constraints. In this study we show however that this type of objective function can also present some non-negligible drawbacks, which will be discussed later in this section.

\footnotetext{
${ }^{7}$ Aside from 16 stations, the line also has 7 stops where passengers can load and unload but no train meets and passes are possible, and 5 tracks which are divided in multiple block sections.
} 
In Table 1 we present results for 5 days of real-life data from the railway line in Stavanger, aggregated over four-hour time periods ${ }^{8}$. A 1 hour time limit was set (and in no instance reached). The objective, agreed with practitioners from the Norwegian infrastructure manager, is of the type shown in Figure 3, with 6 delay intervals (or classes) which are penalized increasingly: less than 1 minute delay, between 1 and 3 , between 3 and 5, between 5 and 10, between 10 and 15 and more than 15 minutes delay. In column 'Periods' we indicate the day and hour range (format: $d d h h-h h$ ). In columns 'Trains' and 'Late' we indicate the average number of trains controlled by the system and the average number of these running late, respectively, within the time period. Column 'Runs' indicates the number of instances considered for the given period in these experiments. ${ }^{9}$ The remaining columns give information about the performance of the two algorithms, namely the average run time ('Time'), the average number of algorithm iterations ('Iterations'), the average time taken to solve each iteration ('It. time'), the average aggregated number of branching nodes made by Cplex in solving each instance ('Branchings') and the average number of initial and generated rows and columns. Note that, for both algorithms, the column 'Iterations' refers to the number of times the algorithm performs the most-time consuming task, namely solving the MILP at hand with Cplex. For $B M$, basically all the overall computation time is taken up by this task. For $P C$, the proportion of time used by Cplex w.r.t. to the other steps of the algorithm (namely 3, 4 and 5, see Section 4) is less skewed but still very significant. Notably, this proportion grows with the size of the problem and number of iterations (an overview is given in Figure 7). Column 'Branchings' in Table 1 is calculated by summing the number of branching nodes explored by Cplex each time it is called during an algorithm run.

As can be noted in Table 1, $P C$ performs better than $B M$ in terms of computation time in 22 periods out of 25 ('Time'). Column 'It. time' shows that the time required by $P C$ for each iteration is, on average, somewhere between $15 \%$ and $30 \%$ of the corresponding iteration time for $B M$. The average number of iterations for $P C$ is (quite naturally) always higher than $B M$, due to the additional micro-iterations that have to be performed by $P C$. Indeed, as described in Section $4, P C$ requires a series of steps (namely 3, 4 and 5) to check for violated cycle and path inequalities. Whenever at least one such inequality is violated, the corresponding constraint is separated and added to the MILP, and Cplex is invoked to find a new optimal selection $y^{k} . P C$ spends a nonnegligible amount of time to perform these steps (depending on the instance, between 5 and 9 out of 10 iterations are of this kind). Therefore, using strategies to skip at least a part of these micro-iterations (e.g. by smart heuristic generation of constraints and variables) could further increase the improvement over $B M{ }^{10}$

Thus, to summarize the performance of the two algorithms: a $P C$ run solves a

\footnotetext{
${ }^{8}$ Note that the data is spread out over 6 days, and that time periods where on average less than 1 train is late were removed

${ }^{9}$ Note that here the instances were randomly sampled at a rate of between 1 and 2 per minute, while, in the real-life setting, on average an instance every 20 seconds has to be solved.

${ }^{10}$ These questions will be matter of future studies.
} 


\begin{tabular}{|c|c|c|c|c|c|c|c|c|c|c|c|c|c|c|c|c|c|c|c|}
\hline \multirow[t]{3}{*}{ Period } & \multirow[t]{3}{*}{ Trains } & \multirow[t]{3}{*}{ Late } & \multirow[t]{3}{*}{ Runs } & \multirow{2}{*}{\multicolumn{2}{|c|}{$\begin{array}{c}\text { Time } \\
\text { (seconds) }\end{array}$}} & \multirow{2}{*}{\multicolumn{2}{|c|}{ Iterations }} & \multirow{2}{*}{\multicolumn{2}{|c|}{$\begin{array}{c}\text { It. time } \\
\text { (milliseconds) }\end{array}$}} & \multirow{2}{*}{\multicolumn{2}{|c|}{ Branchings }} & \multicolumn{4}{|c|}{ Initial } & \multicolumn{4}{|c|}{ Generated } \\
\hline & & & & & & & & & & & & \multicolumn{2}{|c|}{ Rows } & \multicolumn{2}{|c|}{ Columns } & \multicolumn{2}{|c|}{ Rows } & \multicolumn{2}{|c|}{ Columns } \\
\hline & & & & "BM & $\mathrm{PC}$ & $\mathrm{BM}$ & $\mathrm{PC}$ & $\overline{\mathrm{BM}}$ & $\mathrm{PC}$ & $\mathrm{BM}$ & $\mathrm{PC}$ & BM & $\mathrm{PC}$ & 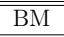 & $\mathrm{PC}$ & $\overline{\mathrm{BM}}$ & $\mathrm{PC}$ & $\mathrm{BM}$ & $\mathrm{PC}$ \\
\hline $112-16$ & 84 & 4 & 216 & 8.01 & 8.87 & 12 & 44 & 691 & 202 & "7258 & 2204 & 3858 & 0 & 3233 & 0 & 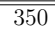 & 822 & 97 & 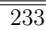 \\
\hline $116-20$ & 62 & 5 & 240 & 2.25 & 1.60 & 6 & 12 & 381 & 133 & 794 & 5 & 2766 & 0 & 2402 & 0 & 372 & 226 & 125 & 135 \\
\hline $120-24$ & 66 & 5 & 240 & 2.17 & 1.32 & 7 & 9 & 314 & 147 & 55 & 0 & 2911 & 0 & 2527 & 0 & 691 & 173 & 256 & 128 \\
\hline $204-08$ & 115 & 7 & 222 & 26.77 & 23.84 & 16 & 117 & 1705 & 204 & 84009 & 11180 & 5044 & 0 & 4263 & 0 & 1590 & 2621 & 401 & 399 \\
\hline $208-12$ & 109 & 5 & 231 & 12.41 & 12.55 & 13 & 54 & 933 & 232 & 14267 & 1296 & 4824 & 0 & 4060 & 0 & 785 & 972 & 217 & 319 \\
\hline $212-16$ & 84 & 3 & 240 & 7.74 & 13.36 & 12 & 60 & 650 & 223 & 4007 & 794 & 3874 & 0 & 3249 & 0 & 425 & 1440 & 115 & 261 \\
\hline $216-20$ & 62 & 4 & 240 & 2.05 & 1.38 & 7 & 10 & 315 & 138 & 206 & 0 & 2758 & 0 & 2395 & 0 & 366 & 185 & 124 & 132 \\
\hline $220-24$ & 66 & 5 & 224 & 6.22 & 3.87 & 9 & 21 & 723 & 184 & 6844 & 574 & 2889 & 0 & 2509 & 0 & 780 & 367 & 276 & 165 \\
\hline $304-08$ & 113 & 3 & 240 & 5.20 & 3.60 & 10 & 19 & 536 & 189 & 912 & 65 & 4957 & 0 & 4164 & 0 & 478 & 379 & 165 & 215 \\
\hline $308-12$ & 105 & 4 & 163 & 2.27 & 1.74 & 7 & 10 & 329 & 174 & 270 & 14 & 4696 & 0 & 3924 & 0 & 84 & 197 & 29 & 155 \\
\hline $312-16$ & 86 & 2 & 240 & 3.16 & 2.15 & 8 & 12 & 405 & 179 & 156 & 0 & 3963 & 0 & 3318 & 0 & 117 & 226 & 51 & 170 \\
\hline 3 16-20 & 57 & 4 & 238 & 2.49 & 1.83 & 6 & 14 & 422 & 131 & 633 & 0 & 2562 & 0 & 2208 & 0 & 198 & 202 & 62 & 133 \\
\hline $320-24$ & 38 & 3 & 240 & 1.21 & 0.34 & 5 & 7 & 257 & 49 & 22 & 0 & 1836 & 0 & 1615 & 0 & 228 & 91 & 84 & 70 \\
\hline $408-12$ & 40 & 1 & 240 & 0.77 & 0.24 & 4 & 5 & 183 & 48 & 4 & 0 & 2338 & 0 & 1945 & 0 & 63 & 68 & 24 & 60 \\
\hline $412-16$ & 30 & 1 & 240 & 0.72 & 0.10 & 4 & 4 & 200 & 25 & 17 & 0 & 1738 & 0 & 1444 & 0 & 77 & 49 & 29 & 45 \\
\hline 4 16-20 & 23 & 1 & 237 & 0.39 & 0.04 & 3 & 3 & 134 & 13 & 2 & 0 & 1180 & 0 & 1000 & 0 & 74 & 36 & 29 & 32 \\
\hline $420-24$ & 15 & 1 & 240 & 0.05 & 0.01 & 2 & 1 & 28 & 10 & 0 & 0 & 745 & 0 & 649 & 0 & 2 & 19 & 1 & 17 \\
\hline $508-12$ & 37 & 1 & 240 & 0.76 & 0.24 & 4 & 6 & 205 & 40 & 28 & 0 & 2346 & 0 & 1976 & 0 & 50 & 63 & 15 & 55 \\
\hline $512-16$ & 34 & 1 & 240 & 1.76 & 0.52 & 6 & 11 & 289 & 47 & 80 & 0 & 2128 & 0 & 1798 & 0 & 89 & 94 & 32 & 71 \\
\hline 5 16-20 & 35 & 2 & 240 & 1.70 & 0.86 & 5 & 11 & 327 & 78 & 81 & 0 & 1875 & 0 & 1628 & 0 & 181 & 108 & 59 & 76 \\
\hline $520-24$ & 59 & 3 & 240 & 3.21 & 1.50 & 7 & 14 & 459 & 107 & 236 & 0 & 2694 & 0 & 2316 & 0 & 318 & 156 & 107 & 115 \\
\hline $604-08$ & 112 & 3 & 239 & 4.58 & 3.30 & 9 & 18 & 492 & 183 & 657 & 39 & 4923 & 0 & 4125 & 0 & 263 & 330 & 84 & 203 \\
\hline $\begin{array}{ll}6 & 08-12 \\
\end{array}$ & 111 & 3 & 107 & 13.25 & 10.89 & 14 & 48 & 946 & 227 & 8032 & 716 & 4843 & 0 & 4053 & 0 & 350 & 804 & 106 & 292 \\
\hline
\end{tabular}

Table 1: Algorithmic information for the convex objective function

significantly higher number of MILPs very fast, while $B M$ requires solving fewer MILPs at each run but is slower. Accordingly, the number of branchings is higher for $B M$ in all periods, while for $P C$ in most periods Cplex is able to solve at root node. In addition, $P C$ always terminates with a significantly smaller MILP (see columns 'Initial' and 'Generated' variables and constraints).

Figure 8 shows the frequency of instances in Table 1 solved within certain time ranges by the two algorithms. The first two columns show how many instances were solved within 1 second, the second two columns between 1 second and 3 seconds and so on. Figure 9 shows the cumulative distribution of the instances solved by the two algorithms, i.e. the percentage of instances solved within a given time. For instance, we notice that $P C$ solves $50 \%$ of instances within 1 second and $72 \%$ of instances within 2 seconds, against $33 \%$ and $56 \%$ for $B M$, respectively. $P C$ reaches the $90 \%$ mark of solved instances after approximately 7 seconds, while $B M$ requires around 9.4 seconds. The chart is truncated at $90 \%$ due to the outliers in the remaining $10 \%$ that hinder its readability.

In Table 2 we present aggregated results with respect to computation time for the same 5 days of data from March 2014. For results presented in this table however, we use a much more stringent time limit for the algorithms, one that is in line with what happens in the practical setting for train traffic management (and other realtime applications). The time limit in this case was set to 30 seconds, as agreed with dispatchers in Stavanger. ${ }^{11}$ Column 'Day' indicates the day of the month the instances were generated. Column 'Runs' indicates the number of instances taken into account

\footnotetext{
${ }^{11}$ Depending on the line this time limit may actually range from a few seconds to minutes.
} 


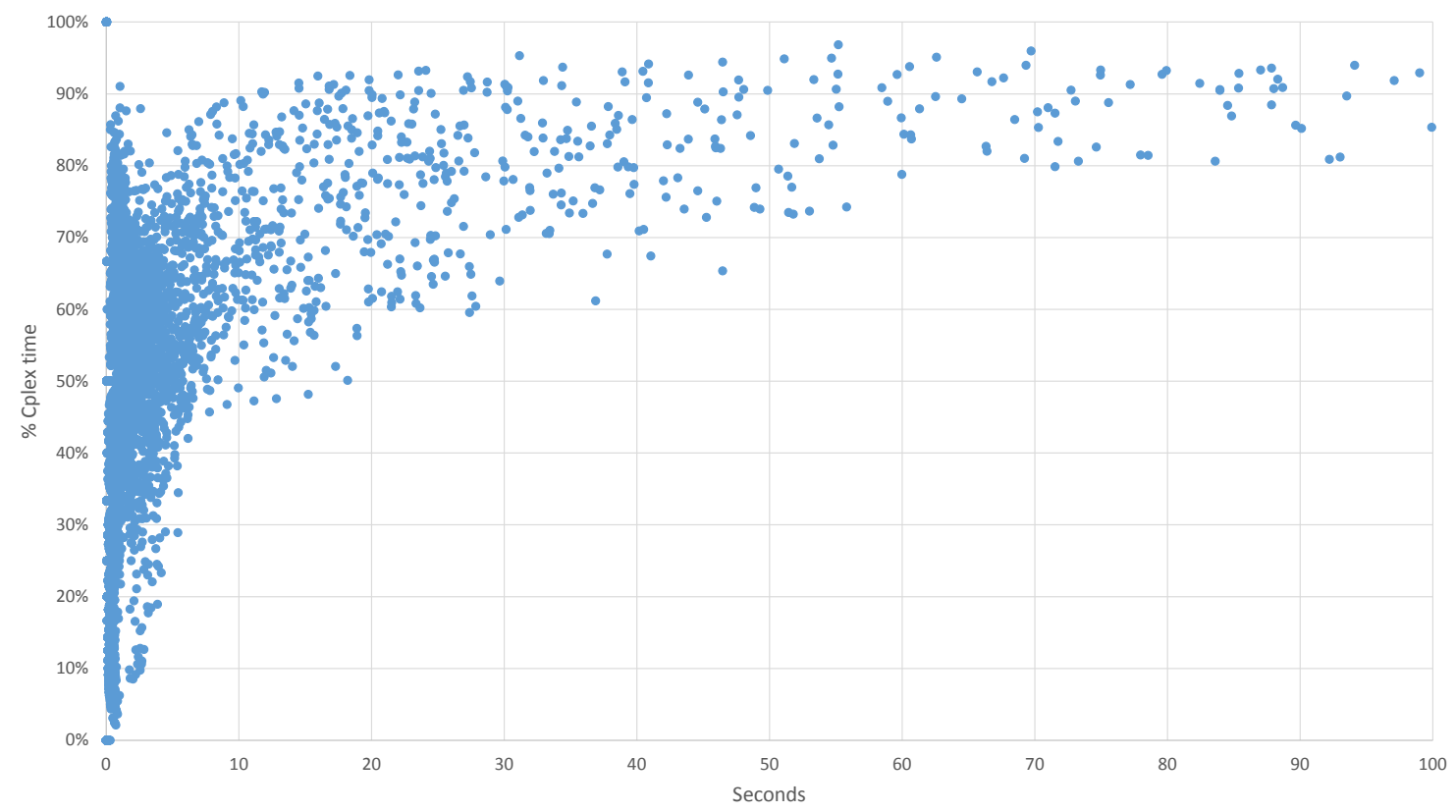

Figure 7: Instances solved by $P C$ represented in terms of overall solution time (horizontal axis) and percentage of solution time used by Cplex respect to the overall solution time (vertical axis).

in this experiment for that day. 'Optimal (\%)' expresses the percentage of instances solved in seconds. We note that the instances for which the optimal solution was not found within the time limit are also counted in the average run time as being solved in 30 seconds. This models what happens in the real-life setting, where the algorithm is given $T$ seconds (in this case $T=30$ ) to find a solution, before some other solution method must come in play to find a solution (a fast, possibly sub-optimal heuristic method or "manual" intervention by the operators). Table 2 shows that on average $P C$ solves faster than $B M$ in all ranges. While the speed up is small for days 1 and 2 (1.07x and $1.01 x$, respectively), it increases to as much as $4.45 x$ on day 4 .

In Table 3 we compare the algorithms on 10 representative instances. These were selected by dividing all our instances in two classes, one with less trains $(<100)$ and one more trains $(>=100)$ and picking 5 at random from each class, with a higher probability placed on instances with more trains late. These instances are available in anonymised form as part of this paper's online companion in Operations Research. In this test, the 1 hour time out (t.o.) was set. In addition to $B M$ and $P C$, we attempt to solve the instances using the full big- $M$ formulation ${ }^{12}$ and feeding the resulting program to Cplex. We denote this approach as FBM. For the columns we use the same headings as in

\footnotetext{
${ }^{12}$ The big- $M$ formulation without using the delayed generation approach, i.e. with all variables and constraints that model the possible disjunctive pairs.
} 


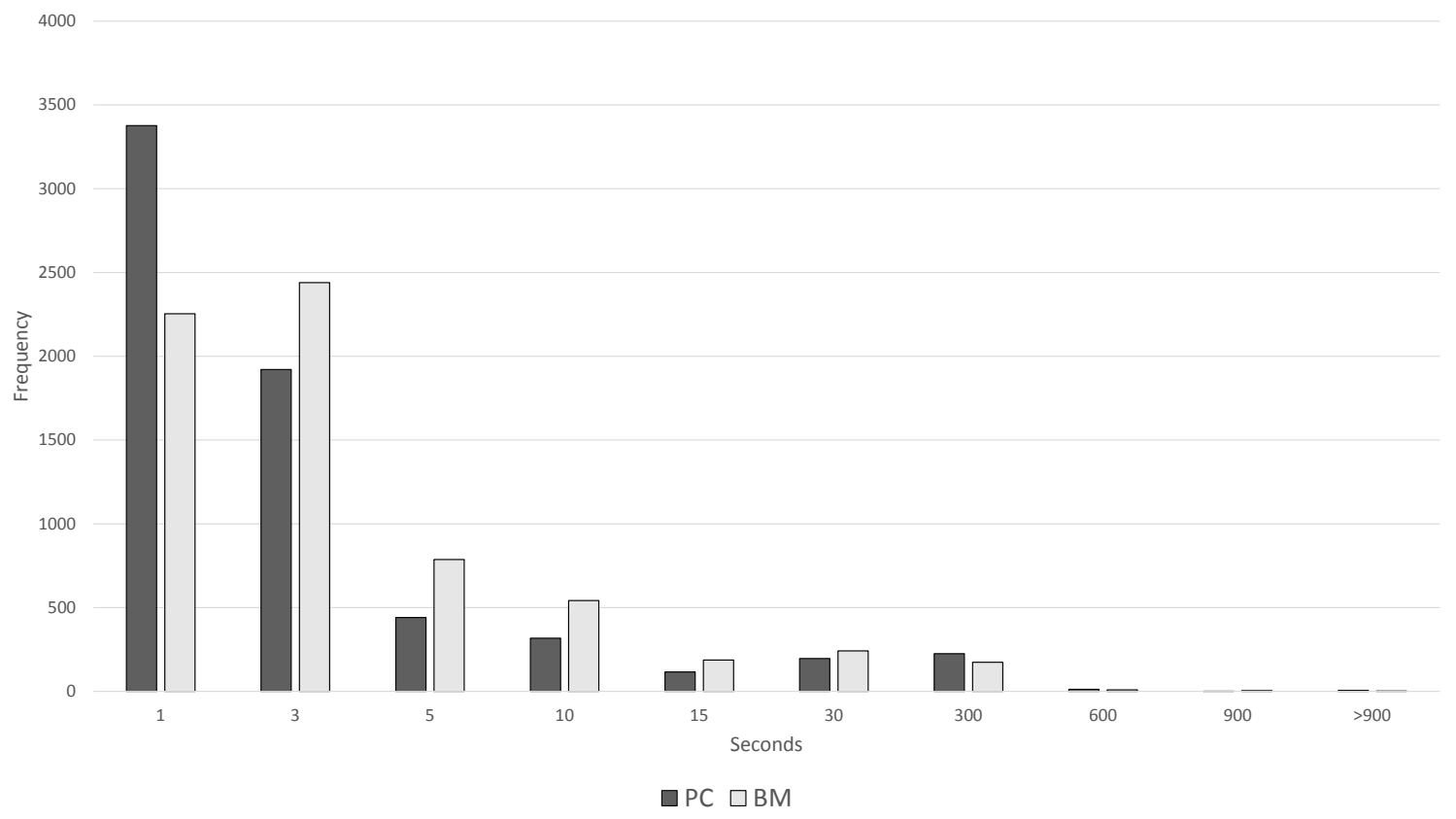

Figure 8: Histogram of the frequency of instances with respect to computational time presented in Table 1.

Table 1, with the exception of 'Last it. time' (which expresses the time taken by Cplex to solve the last MILP) and 'Rows' and 'Columns' (which here represent the total number of rows and column of the final MILP, i.e. initial plus generated). Note that for $F B M$ we omit columns 'Iterations' and 'Last it.time' as there is no delayed generation, thus it solves a single MILP/iteration. In column 'Time' we notice how FBM takes orders of magnitude more computation time respect to the other two approaches, timing out on 3 occasions (more than 1 hour running). Moreover, again for $F B M$, the 32-bit version of Cplex crashed in solving instances 5 to 10 due to lack of memory, requiring the use of the 64-bit version. This results clearly demonstrate that solving "directly" the full big- $M$ would be impracticable, at least for our test-bed of real-life traffic management instances. As shown for the aggregate results, $P C$ solves a higher number of MILPs w.r.t. $B M$ but spending significantly less time. This can be seen as consequence of the smaller size of the MILPs (see 'Rows' and 'Columns') and more importantly of the stronger formulation which allows to explore a reduced search tree (see 'Branchings'). In addition, the time taken to solve the last iteration/MILP is considerably smaller for $P C$, again pointing to the fact that, with an effective strategy to generate the "right" constraints and variables (and skipping as many micro-iterations as possible), one could further improve the overall performance of the algorithm.

Convex functions are, in many cases, a reasonable proxy of a planner's decision process. However, in many cases the effect on decisions can become increasingly distant 


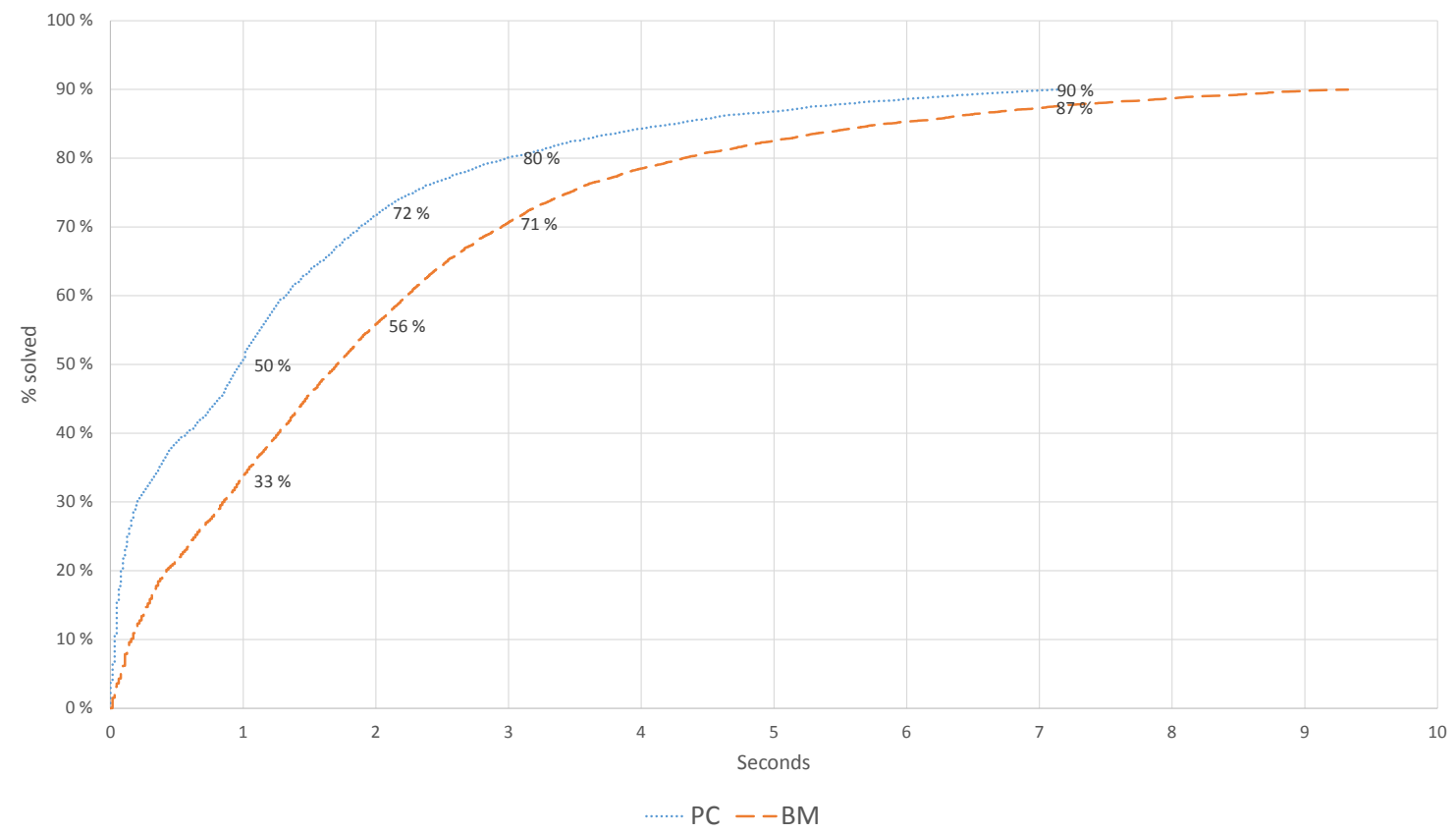

Figure 9: Cumulative distribution of the percentage of instances solved in Table 1 with respect to computation time.

from the wish or behaviour of the planners, operators, stakeholders. We will elaborate on this more in the following subsection.

\subsection{Non-convex non-decreasing (non-negative) cost functions}

A class of cost functions frequently adopted by practitioners in traffic management problems, is that of non-convex non-decreasing (non-negative) functions. In many cases, these functions model the recovery strategies of planners more accurately than convex ones. In public transport for instance, heavily delayed vehicles often actually lose priority over other vehicles after a certain threshold (the opposite of what the corresponding convex objective function would enforce). Controllers, dispatchers, or the operators themselves, may prefer avoiding the recovery of such a vehicle should it come at the price of delaying others. In other words, they may favour the strategy of not further disturbing the system, rather than attempting the recovery of one (or more) heavily delayed vehicles. In mathematical terms, this can be expressed with a function of the type depicted in Figure 10.

The "loss of convexity" is generally seen as a drawback, as the computational complexity of traditional models tends to increase as a consequence (e.g. for $B M$ a function of this kind requires adding suitable binary variables and constraints).

In the following we compare the two algorithms using a non-convex non-decreasing 


\begin{tabular}{|c|c|c|c|c|c|c|}
\hline \multirow[t]{2}{*}{ Day } & \multirow[t]{2}{*}{ Runs } & \multicolumn{2}{|c|}{ Optimal (\%) } & \multicolumn{2}{|c|}{ Run time (s) } & \multirow[t]{2}{*}{ Speed up } \\
\hline & & $\mathrm{BM}$ & $\mathrm{PC}$ & BM & $\mathrm{PC}$ & \\
\hline 1 & 696 & 99.6 & 97.6 & 4.01 & 3.76 & $1.07 \mathrm{x}$ \\
\hline 2 & 1188 & 88.6 & 82.9 & 10.93 & 10.85 & $1.01 \mathrm{x}$ \\
\hline 3 & 1360 & 100 & 100 & 2.92 & 1.86 & $1.57 \mathrm{x}$ \\
\hline 4 & 1434 & 100 & 100 & 0.49 & 0.11 & $4.45 \mathrm{x}$ \\
\hline 5 & 1378 & 100 & 100 & 1.34 & 0.55 & $2.44 \mathrm{x}$ \\
\hline
\end{tabular}

Table 2: Aggregate comparisons for 5 days of computations using the convex objective function.

\begin{tabular}{|c|c|c|c|c|c|c|c|c|c|c|c|c|c|c|c|c|c|c|}
\hline \multirow[t]{2}{*}{ Id } & \multirow[t]{2}{*}{ Trains } & \multirow[t]{2}{*}{ Late } & \multicolumn{3}{|c|}{$\begin{array}{c}\text { Time } \\
\text { (seconds) }\end{array}$} & \multicolumn{2}{|c|}{ Iterations } & \multicolumn{2}{|c|}{$\begin{array}{l}\text { Last it. time } \\
\text { (milliseconds) }\end{array}$} & \multicolumn{3}{|c|}{ Branchings } & \multicolumn{3}{|c|}{ Rows } & \multicolumn{3}{|c|}{ Columns } \\
\hline & & & FBM & $\mathrm{BM}$ & $\mathrm{PC}$ & $\mathrm{BM}$ & $\mathrm{PC}$ & BM & $\mathrm{PC}$ & FBM & $\mathrm{BM}$ & $\mathrm{PC}$ & FBM & $\mathrm{BM}$ & $\mathrm{PC}$ & FBM & BM & $\mathrm{PC}$ \\
\hline 1 & 77 & 0 & 88.34 & 0.41 & 0.15 & 2 & 2 & 200 & 23 & 443 & 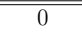 & 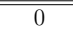 & 126361 & 2912 & 84 & 14562 & 2858 & 81 \\
\hline 2 & 96 & 2 & 276.72 & 13.80 & 3.88 & 14 & 29 & 620 & 30 & 1627 & 1723 & 0 & 203894 & 4669 & 500 & 22658 & 3815 & 242 \\
\hline 3 & 96 & 1 & 196.46 & 6.77 & 6.16 & 14 & 31 & 1654 & 21 & 903 & 1700 & 0 & 201583 & 4678 & 525 & 22487 & 3812 & 240 \\
\hline 4 & 92 & 3 & 204.01 & 11.25 & 2.71 & 14 & 34 & 942 & 154 & 1129 & 3561 & 0 & 182640 & 4555 & 640 & 20696 & 3660 & 260 \\
\hline 5 & 88 & 4 & 238.35 & 6.77 & 6.31 & 9 & 32 & 901 & 334 & 1585 & 2699 & 0 & 171706 & 4332 & 520 & 19706 & 3534 & 230 \\
\hline 6 & 105 & 3 & 549.97 & 42.92 & 22.61 & 17 & 101 & 3846 & 540 & 5398 & 24517 & 2184 & 223957 & 5962 & 2174 & 23910 & 4358 & 341 \\
\hline 7 & 105 & 3 & 2025.69 & 137.68 & 88.71 & 18 & 175 & 30018 & 736 & 27691 & 115475 & 26258 & 229178 & 6477 & 3493 & 28035 & 4421 & 407 \\
\hline 8 & 119 & 7 & t.o. & 96.68 & 78.37 & 20 & 136 & 18037 & 513 & - & 84144 & 7267 & 290091 & 7088 & 3609 & 35173 & 4920 & 493 \\
\hline 9 & 118 & 5 & t.o. & 95.39 & 117.55 & 19 & 180 & 38582 & 615 & - & 86802 & 15493 & 286200 & 6981 & 3642 & 34600 & 4854 & 518 \\
\hline 10 & 120 & 7 & t.o. & 510.96 & 373.24 & 20 & 229 & 51564 & 5758 & - & 606519 & 36170 & 288642 & 6884 & 7008 & 34812 & 4885 & 522 \\
\hline
\end{tabular}

Table 3: Algorithmic information for 10 representative instances with the convex objective function

(non-negative) objective function. In particular, we use a cost function with a similar structure to that used for the convex case (the same 6 delay intervals), only with a "plateau" for the last interval (more than 15 minutes delay). In other words, any train delayed more than 15 minutes, whose delay cannot be brought under such threshold, loses all dispatching priority. Comparisons are presented in Tables 4, 5 and 6, for the same instances and indicators used previously in Tables 1, 2 and 3, respectively.

Table 4 confirms and adds to the results obtained with the convex objective. Indeed, the trade-off between shorter (average) iteration time and higher number of iterations is even more favourable to $P C$ in this case, as it performs better than $B M$ in terms of average computation time in 23 periods out of 25 . This improvement can be explained as the consequence of $P C$ solving even smaller MILPs than in the convex case, which can be noted by the size of the MILPs (columns 'Initial' + 'Generated') and the number of branchings ( $P C$ on average solves every sub-problem at node 0 for 19 periods of 25).

Aside from $P C$ performing proportionally better with this objective than $B M$, an improvement w.r.t to the convex case was registered for both algorithms, which may seem counter-intuitive given the increased computational complexity of using such a non-convex objective. However, this can be explained intuitively analyzing the behaviour of the algorithms in situations of disruption. Indeed, in the convex case, the number of "similar" solutions (in terms of objective function) is often very high. There- 


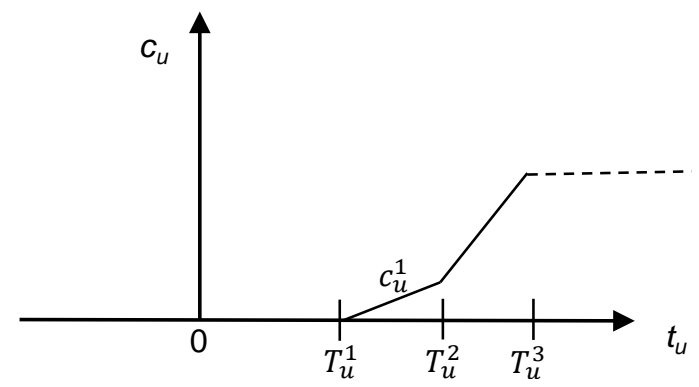

Figure 10: Example of non-convex non-decreasing (non-negative) function.

fore algorithms such as $B M$ and $P C$ that follow a "delayed" approach will tend to generate many rows and columns associated with potential conflicts to explore such solutions.

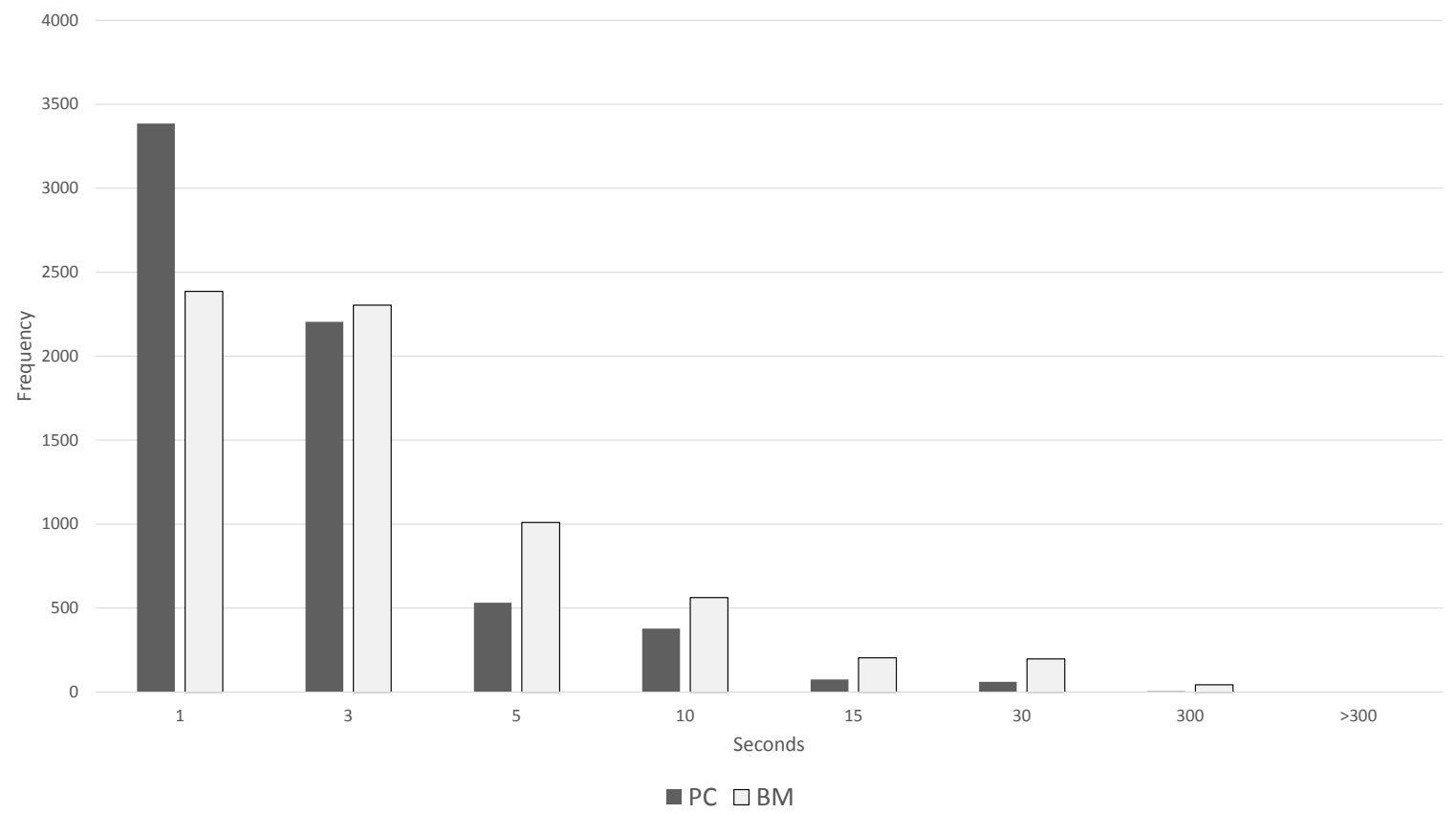

Figure 11: Histogram of the frequency with respect to computational time for instances in Table 4.

In Figure 11 we show the frequency of instances in Table 4 solved within certain time ranges. The superior performance of $P C$ in terms of computation time stands out as $P C$ has less instances in each range except for the less than 1 second case. Similarly, in Figure 12 we show that $P C$ solves a higher percentage of instances than $B M$ by any given computation time. For example, $P C$ solves $50 \%$ of the instances within 1 second, 


\begin{tabular}{|c|c|c|c|c|c|c|c|c|c|c|c|c|c|c|c|c|c|c|c|}
\hline \multirow[t]{3}{*}{ Period } & \multirow[t]{3}{*}{ Trains } & \multirow[t]{3}{*}{ Late } & \multirow[t]{3}{*}{ Runs } & \multirow{2}{*}{\multicolumn{2}{|c|}{$\begin{array}{c}\text { Time } \\
\text { (seconds) }\end{array}$}} & \multirow{2}{*}{\multicolumn{2}{|c|}{ Iterations }} & \multirow{2}{*}{\multicolumn{2}{|c|}{$\begin{array}{c}\text { It. time } \\
\text { (milliseconds) }\end{array}$}} & \multirow{2}{*}{\multicolumn{2}{|c|}{ Branchings }} & \multicolumn{4}{|c|}{ Initial } & \multicolumn{4}{|c|}{ Generated } \\
\hline & & & & & & & & & & & & \multicolumn{2}{|c|}{ Rows } & \multicolumn{2}{|c|}{ Columns } & \multicolumn{2}{|c|}{ Rows } & \multicolumn{2}{|c|}{ Columns } \\
\hline & & & & BM & $\mathrm{PC}$ & BM & $\mathrm{PC}$ & BM & $\mathrm{PC}$ & BM & $\mathrm{PC}$ & BM & PC & BM & $\overline{\mathrm{PC}}$ & BM & $\mathrm{PC}$ & BM & $\mathrm{PC}$ \\
\hline $112-16$ & 84 & 4 & 216 & 4.75 & 6.35 & 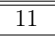 & 31 & 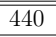 & 207 & 2904 & 172 & 3941 & 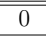 & 3233 & 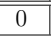 & 324 & ב569 & 993 & 233 \\
\hline $116-20$ & 62 & 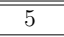 & 240 & 2.09 & 1.69 & 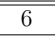 & 12 & 327 & 147 & 247 & 0 & 2827 & (20 & 2402 & "0 & 383 & 222 & 127 & 135 \\
\hline $120-24$ & 66 & (5 & 240 & 3.53 & 1.30 & 7 & 9 & 527 & 141 & 126 & "0 & 2977 & 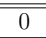 & 2527 & "0 & 698 & 173 & 260 & 128 \\
\hline $204-08$ & 115 & 7 & 222 & 17.77 & 4.77 & 21 & 18 & 858 & 273 & 2708 & 0 & 5160 & 0 & $\begin{array}{l}4263 \\
\end{array}$ & 0 & 2751 & 4420 & 614 & 310 \\
\hline $208-12$ & 109 & 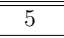 & 231 & 11.49 & 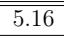 & 15 & 21 & 787 & 246 & 4067 & 12 & 49933 & 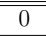 & ב4060 & 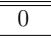 & 1393 & 4434 & 320 & 278 \\
\hline $212-16$ & 84 & 3 & 240 & $\begin{array}{c}6.76 \\
\end{array}$ & 5.14 & 11 & 25 & (2609 & 210 & 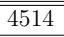 & 207 & 3958 & 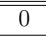 & 3249 & 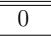 & 809 & 4466 & 156 & 212 \\
\hline 2 216-20 & 62 & 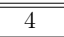 & 240 & 2.20 & 1.26 & 7 & 10 & ב333 & 125 & 94 & 0 & 2819 & $\overline{0}$ & 2395 & 0 & 376 & 184 & $\bar{~} 124$ & 131 \\
\hline $220-24$ & 266 & 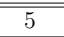 & 224 & 4.94 & 2.90 & 8 & 16 & "633 & 177 & 5307 & 32 & 2955 & 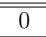 & 2509 & 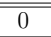 & 780 & 276 & 275 & 161 \\
\hline $\begin{array}{l}304-08 \\
\end{array}$ & 1113 & 3 & 240 & $\begin{array}{c}4.17 \\
\end{array}$ & 2.22 & 8 & 12 & 502 & 193 & 666 & 0 & 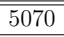 & (20 & 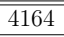 & 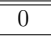 & 881 & 252 & 2220 & 198 \\
\hline $308-12$ & 105 & 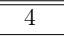 & 163 & 2.66 & $\begin{array}{l}1.76 \\
\end{array}$ & 6 & 10 & "416 & 181 & 259 & 111 & 48801 & (20 & 3924 & " 0 & 101 & 197 & 39 & 154 \\
\hline $312-16$ & 86 & 2 & 240 & 2.97 & 2.15 & 8 & 12 & 386 & 173 & 477 & 0 & 4050 & 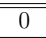 & 3318 & 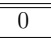 & 142 & 227 & 58 & 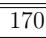 \\
\hline 3 16-20 & (57 & 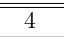 & 238 & 2.16 & 1.83 & 6 & 14 & 338 & 133 & 417 & 0 & 2615 & 0 & 2208 & 0 & 216 & 202 & 66 & 133 \\
\hline $320-24$ & 38 & 3 & 240 & 1.01 & $\begin{array}{c}0.34 \\
\end{array}$ & 5 & 7 & 210 & (250 & 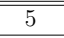 & 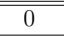 & 1874 & 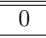 & 1615 & 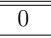 & 235 & 991 & 88 & 70 \\
\hline $\begin{array}{c}408-12 \\
\end{array}$ & 40 & 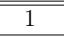 & 240 & $\begin{array}{ll}1.04 \\
\end{array}$ & ב0.24 & 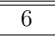 & 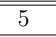 & 182 & 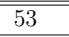 & 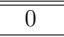 & 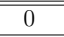 & 2378 & 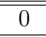 & 1945 & 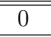 & 104 & 68 & 32 & 60 \\
\hline 4 12-16 & 30 & $\overline{1}$ & 240 & 0.44 & 0.10 & 4 & 4 & 119 & 29 & 1 & 0 & 1768 & 0 & 1444 & 0 & 81 & 49 & 31 & 45 \\
\hline 4 4 16-20 & 23 & 1 & 237 & 0.32 & 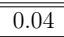 & 3 & 3 & 107 & 14 & 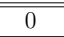 & 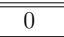 & 1203 & 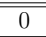 & 1000 & 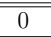 & 75 & 35 & 29 & 32 \\
\hline $\begin{array}{l}420-24 \\
\end{array}$ & 15 & 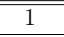 & 240 & 0.05 & 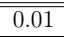 & 2 & 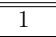 & 28 & 99 & 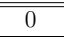 & 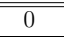 & 7670 & 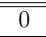 & 649 & 0 & 2 & 10 & 1 & 8 \\
\hline $\begin{array}{l}508-12 \\
\end{array}$ & 37 & 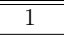 & 240 & "0.77 & "0.23 & 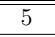 & 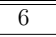 & 1711 & 40 & 15 & " 0 & 2383 & 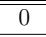 & 1976 & "0 & 55 & "63 & 18 & 55 \\
\hline $\begin{array}{l}512-16 \\
\end{array}$ & 34 & 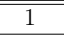 & 240 & 0.97 & "0.52 & 6 & 111 & 156 & 47 & 10 & 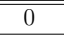 & 2162 & 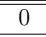 & 17988 & " 0 & 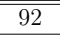 & 994 & 33 & 71 \\
\hline 5 16-20 & 35 & 2 & 240 & 1.07 & 0.87 & 5 & 11 & 214 & 78 & 8 & 0 & 1910 & 0 & 1628 & 0 & 182 & 108 & 59 & 76 \\
\hline $520-24$ & 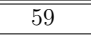 & 3 & 240 & 2.63 & " 1.49 & 6 & 14 & 4244 & 110 & 22 & (20 & 2753 & "0 & 2316 & 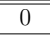 & 318 & 155 & 109 & 115 \\
\hline (204-08 & 1112 & 3 & 239 & 3.73 & 2.32 & 8 & 13 & 4448 & 186 & 85 & 0 & 5035 & 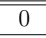 & 4125 & 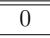 & "2679 & 240 & 142 & 192 \\
\hline $\begin{array}{ll} & 08-12 \\
\end{array}$ & 1111 & 3 & 107 & 10.96 & 12.08 & 14 & 50 & 766 & 242 & 97924 & 1028 & 49954 & 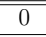 & 4053 & 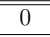 & 399 & 845 & 1114 & 295 \\
\hline
\end{tabular}

Table 4: Algorithmic information for the non-convex objective function.

$74 \%$ after 2 seconds, $94 \%$ after 5 seconds and $98 \%$ after 10 seconds, against the 35\%, $56 \%, 85 \%$ and $94 \%$ of $B M$, respectively.

\begin{tabular}{||c||c||c|c||c|c||c||}
\hline \multicolumn{1}{||c||}{ Day } & \multicolumn{1}{|c||}{ Runs } & \multicolumn{2}{c||}{ Optimal (\%) } & \multicolumn{2}{c||}{ Run time (s) } & Speed up \\
& & BM & PC & BM & PC & \\
\hline \hline 26 & 696 & $99.9 \%$ & $99.9 \%$ & 3.41 & 3.00 & $1.14 \mathrm{x}$ \\
\hline 27 & 1188 & $97 \%$ & $100 \%$ & 8.47 & 3.79 & $2.23 \mathrm{x}$ \\
\hline 28 & 1360 & $100 \%$ & $100 \%$ & 2.78 & 1.62 & $1.72 \mathrm{x}$ \\
\hline 29 & 1434 & $100 \%$ & $100 \%$ & 0.52 & 0.10 & $5.20 \mathrm{x}$ \\
\hline 30 & 1378 & $100 \%$ & $100 \%$ & 0.94 & 0.55 & $1.71 \mathrm{x}$ \\
\hline
\end{tabular}

Table 5: Aggregate comparisons for 5 days of computations using the non-convex function

In Table 5 we show the performance of the algorithms aggregated over each day of instances. Similarly to Table 2, we set a 30 second time limit for the algorithms to find the optimal solution. We notice that the average speed up achieved using $P C$ increases compared to the convex case (Table 2) on the first 4 days. $^{13}$ In particular,

\footnotetext{
${ }^{13} \mathrm{On}$ the last day, the average computation time for $P C$ is actually the same as the convex case (0.55 seconds), while $B M$ performs better (0.94 seconds against 1.34$)$. Still, $P C$ performs better than $B M$ (0.55 against 0.94 seconds).
} 


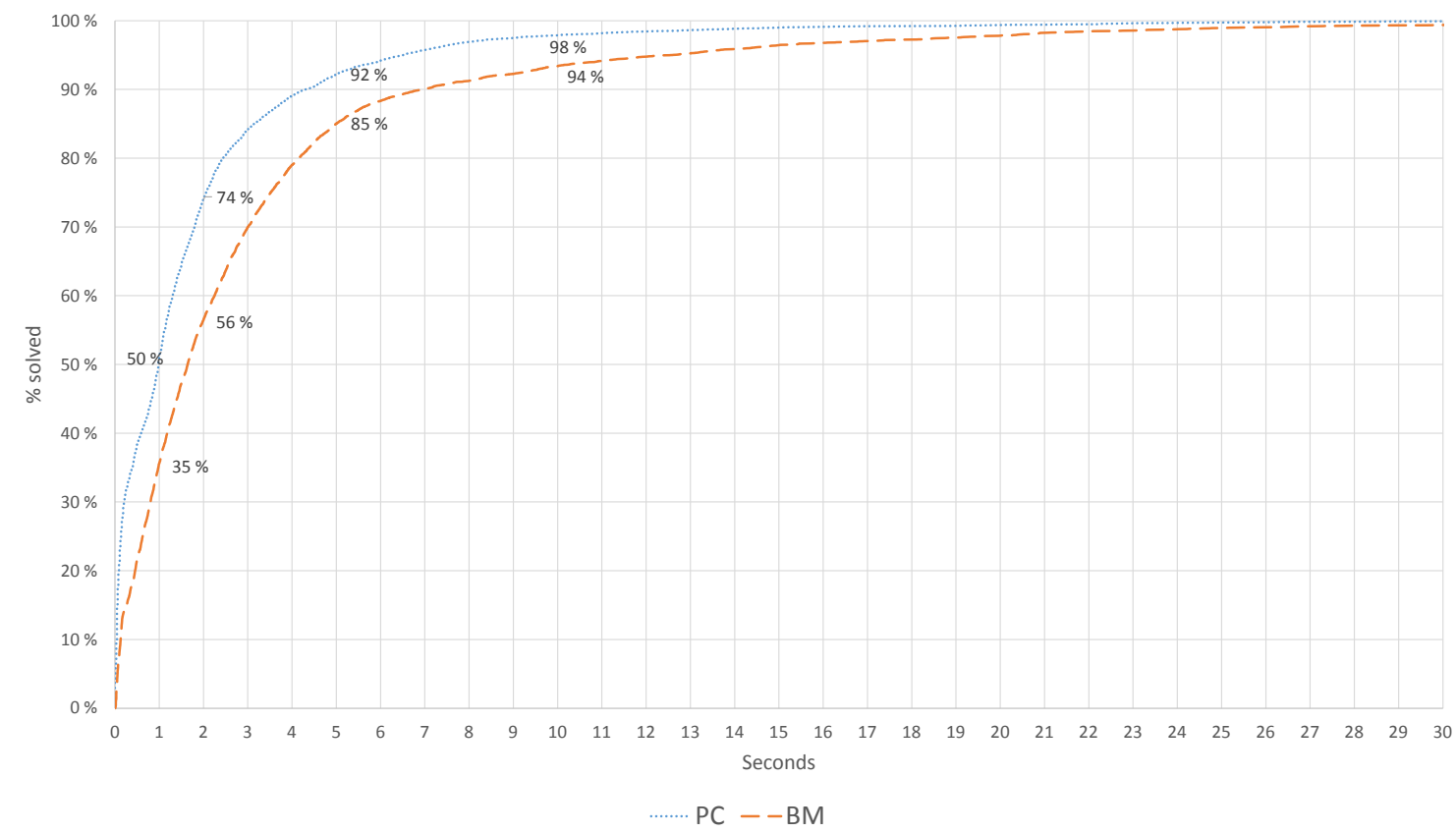

Figure 12: Cumulative distribution of the percentage of instances solved in Table 4 with respect to computation time.

this improvement is most noticeable on day 2, where $P C$ is 2.23 times faster than $B M$ on average, against the convex case where the algorithms basically performed the same (1.01x speed up, see Table 2).

\begin{tabular}{|c|c|c|c|c|c|c|c|c|c|c|c|c|c|c|c|c|c|c|}
\hline \multirow[t]{2}{*}{ Id } & \multirow[t]{2}{*}{ Trains } & \multirow[t]{2}{*}{ Late } & \multicolumn{3}{|c|}{$\begin{array}{c}\text { Time } \\
\text { (seconds) }\end{array}$} & \multicolumn{2}{|c|}{ Iterations } & \multicolumn{2}{|c|}{$\begin{array}{l}\text { Last It. time } \\
\text { (milliseconds) }\end{array}$} & \multicolumn{3}{|c|}{ Branchings } & \multicolumn{3}{|c|}{ Rows } & \multicolumn{3}{|c|}{ Columns } \\
\hline & & & "FBM & BM & $\mathrm{PC}$ & $\overline{\mathrm{BM}}$ & $\mathrm{PC}$ & 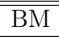 & $\mathrm{PPC}$ & FBM & $\overline{\mathrm{BM}}$ & $\mathrm{PC}$ & FBM & BM & $\mathrm{PC}$ & FBM & $\overline{\mathrm{BM}}$ & $\mathrm{PC}$ \\
\hline 1 & 77 & 0 & 9.35 & 1.03 & 0.07 & 4 & 1 & 162 & 20 & 0 & 0 & 0 & 126438 & 2999 & 84 & 14562 & 2863 & 81 \\
\hline 2 & 96 & 2 & 52.61 & 8.55 & 5.07 & 15 & 28 & 387 & 141 & 281 & 1306 & 0 & 208387 & 4869 & 500 & 26385 & 3824 & 242 \\
\hline 3 & 96 & 1 & 44.56 & 6.94 & 5.61 & 15 & 30 & 570 & 225 & 423 & 1395 & 0 & 206067 & 4868 & 519 & 26207 & 3819 & 240 \\
\hline 4 & 92 & 3 & 33.53 & 4.28 & 7.26 & 14 & 32 & 241 & 140 & 595 & 2163 & 0 & 186921 & 4713 & 628 & 24254 & 3661 & 260 \\
\hline 5 & 88 & 4 & 28.39 & 7.13 & 5.24 & 13 & 29 & 508 & 165 & 283 & 1748 & 0 & 175847 & 4498 & 478 & 23149 & 3535 & 230 \\
\hline 6 & 105 & 3 & 67.42 & 9.47 & 1.69 & 9 & 16 & 730 & 10 & 444 & 0 & 0 & 228616 & 6820 & 307 & 27838 & 4482 & 257 \\
\hline 7 & 105 & 3 & 85.35 & 6.75 & 3.44 & 12 & 16 & 582 & 285 & 411 & 80 & 0 & 229283 & 8387 & 305 & 28035 & 4743 & 267 \\
\hline 8 & 119 & 7 & 3257.91 & 22.03 & 2.29 & 17 & 13 & 1663 & 20 & 9309 & 4408 & 0 & 290144 & 9136 & 610 & 35173 & 5243 & 371 \\
\hline 9 & 118 & 5 & 2301.93 & 29.47 & 2.07 & 14 & 13 & 1795 & 10 & 3475 & 2736 & 0 & 288766 & 9002 & 428 & 34812 & 5193 & 322 \\
\hline 10 & 120 & 7 & t.o. & 22.12 & 1.33 & 14 & 13 & 2754 & 10 & - & 3722 & 0 & & 9366 & 619 & & 5278 & 368 \\
\hline
\end{tabular}

Table 6: Algorithmic information for 10 representative instances with the non-convex objective function

In Table 6 we compare the algorithms on the same 10 representative instances in Table 6 using the non-convex objective. Again, we additionally attempt to solve the instances using the full big- $M$ formulation and feed the resulting program to Cplex (approach FBM). What stands out from Table 6 is that $P C$ performs significantly better than $B M$, in particular for the hard instances 8-10, for which the improvement 
is drastic. Again, in searching for a rationale behind this improvement it is natural to point to the size of the MILPs being solved and the number of branchings required to solve them (significantly smaller than for the big-Mapproaches). Also to be noted is that $F B M$ performs significantly better than in the convex case, at least for some instances, but remains very distant from $B M$ and $P C$ in terms of overall performance.

These experiments somehow confirm the intuitive fact that the choice of objective function, even reasonably similar ones, can non-negligibly impact the performance of an algorithm. Therefore, it is important, particularly when dealing with real-life problems for which the definition of the objective can be fuzzy, to carefully elicitate and analyze the specifications, rules of thumb, wishes and goals of the stakeholders. In some cases, real-life may be simpler than the models. To give an example, the performance of the traffic management department of the Danish train operating company DSB is evaluated on a single indicator, namely the percentage of trains delayed over 3 minutes ([24]). Thus, practically speaking, their only true objective is to comply with this requirement. The resulting (binary) cost function is shown in Figure 13.

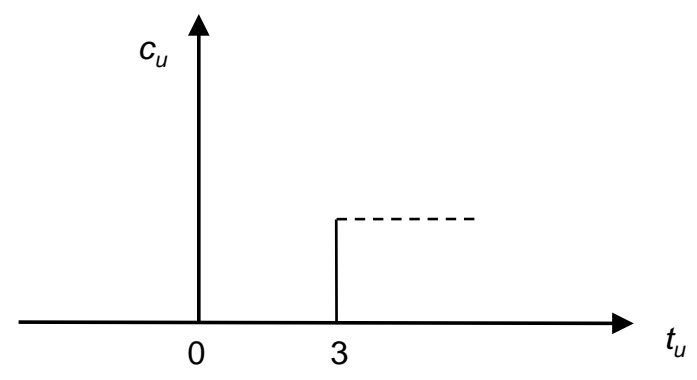

Figure 13: Example of binary function: 3-minutes-or-die.

We finally test and compare the performance of the two algorithms using the DSB cost function in Figure 13. We present the results in analogous Tables to those presented for the previous objectives. In Table 7 we give algorithmic information regarding the solution process when using the binary objective function. In general, both algorithms perform competitively, solving on average under 3 seconds for all time periods. Again however $P C$ performs better than $B M$ in 23 periods out of 25 , "losing" by only decimals of a second in periods $204-08$ and $220-24$.

Like for the previous two objectives, in Figures 14 and 15 we show the frequency of instances being solved within certain time ranges and their cumulative distribution. Again, it can be noted that for any given computation time $P C$ has cumulatively solved more, or at least as many, instances as $B M$. Finally, in Table 8 we show the performance of the algorithms aggregated over each day of instances also for the binary objective. $P C$ performs better than $B M$ on all 5 days, with the performance being very similar on day 2 and noticeably better on all the other days. 


\begin{tabular}{|c|c|c|c|c|c|c|c|c|c|c|c|c|c|c|c|c|c|c|c|}
\hline \multirow[t]{3}{*}{ Period } & \multirow[t]{3}{*}{ Trains } & \multirow[t]{3}{*}{ Late } & \multirow[t]{3}{*}{ Instances } & \multirow{2}{*}{\multicolumn{2}{|c|}{$\begin{array}{c}\text { Run time } \\
\text { (seconds) }\end{array}$}} & \multirow{2}{*}{\multicolumn{2}{|c|}{ Iterations }} & \multirow{2}{*}{\multicolumn{2}{|c|}{$\begin{array}{c}\text { Iteration time } \\
\text { (milliseconds) }\end{array}$}} & \multirow{2}{*}{\multicolumn{2}{|c|}{ Branchings }} & \multicolumn{4}{|c|}{ Initial } & \multicolumn{4}{|c|}{ Generated } \\
\hline & & & & & & & & & & & & \multicolumn{2}{|c|}{ Rows } & \multicolumn{2}{|c|}{ Columns } & \multicolumn{2}{|c|}{ Rows } & \multicolumn{2}{|c|}{ Columns } \\
\hline & & & & BM & $\mathrm{PC}$ & $\overline{\mathrm{BMM}}$ & $\overline{\mathrm{PC}}$ & $\mathrm{BM}$ & $\mathrm{PC}$ & $\overline{\mathrm{BM}}$ & $\overline{P \mathrm{PC}}$ & BM & $\overline{\mathrm{PC}}$ & BM & $\overline{\mathrm{PC}}$ & BM & $\mathrm{PC}$ & $\overline{\mathrm{BM}}$ & $\mathrm{PC}$ \\
\hline $112-16$ & 84 & 4 & 216 & 1.44 & 0.89 & 10 & 19 & 145 & 46 & 65 & 0 & 3944 & 0 & 2900 & 0 & 867 & 476 & 172 & 307 \\
\hline $116-20$ & 62 & 5 & 240 & 0.82 & 0.38 & 8 & 11 & 98 & 33 & 20 & 0 & 2827 & 0 & 2155 & 0 & 784 & 199 & 201 & 204 \\
\hline $120-24$ & 66 & 5 & 240 & 0.62 & 0.55 & 6 & 7 & 100 & 77 & 0 & 0 & 2977 & 0 & 2263 & 0 & 1441 & 134 & 372 & 187 \\
\hline $204-08$ & 115 & 7 & 222 & 2.91 & 3.04 & 10 & 23 & 294 & 135 & 70 & 0 & 5160 & 0 & 3805 & 0 & 3821 & 465 & 653 & 446 \\
\hline $208-12$ & 109 & 5 & 231 & 2.18 & 1.74 & 10 & 17 & 229 & 101 & 7 & 0 & 4933 & 0 & 3623 & 0 & 1574 & 370 & 345 & 388 \\
\hline $212-16$ & 84 & 3 & 240 & 1.07 & 0.96 & 9 & 17 & 126 & 57 & 7 & 0 & 3958 & 0 & 2914 & 0 & 922 & 343 & 184 & 298 \\
\hline $216-20$ & 62 & 4 & 240 & 0.63 & 0.48 & 7 & 10 & 91 & 47 & 3 & 0 & 2819 & 0 & 2149 & 0 & 652 & 166 & 178 & 196 \\
\hline $220-24$ & 66 & 5 & 224 & 0.86 & 0.83 & 7 & 9 & 130 & 91 & 0 & 0 & 2955 & 0 & 2246 & 0 & 1881 & 170 & 384 & 215 \\
\hline $304-08$ & 113 & 3 & 240 & 1.09 & 1.16 & 8 & 9 & 140 & 127 & 1 & 0 & 5070 & 0 & 3713 & 0 & 1415 & 222 & 292 & 313 \\
\hline $308-12$ & 105 & 4 & 163 & 0.74 & 0.47 & 7 & 7 & 104 & 69 & 0 & 0 & 4801 & 0 & 3502 & 0 & 495 & 164 & 114 & 258 \\
\hline 3 12-16 & 86 & 2 & 240 & 0.71 & 0.52 & 8 & 10 & 95 & 53 & 0 & 0 & 4050 & 0 & 2973 & 0 & 470 & 209 & 121 & 259 \\
\hline $316-20$ & 57 & 4 & 238 & 0.75 & 0.40 & 8 & 10 & 91 & 39 & 5 & 0 & 2615 & 0 & 1978 & 0 & 627 & 154 & 140 & 196 \\
\hline $320-24$ & 38 & 3 & 240 & 0.39 & 0.11 & 6 & 5 & 70 & 20 & 0 & 0 & 1874 & 0 & 1464 & 0 & 528 & 77 & 123 & 109 \\
\hline $408-12$ & 40 & 1 & 240 & 0.21 & 0.08 & 5 & 3 & 45 & 24 & 0 & 0 & 2378 & 0 & 1785 & 0 & 163 & 60 & 54 & 100 \\
\hline $412-16$ & 30 & 1 & 240 & 0.29 & 0.04 & 5 & 3 & 58 & 13 & 0 & 0 & 1768 & 0 & 1322 & 0 & 123 & 45 & 45 & 75 \\
\hline 4 16-20 & 23 & 1 & 237 & 0.30 & 0.02 & 6 & 2 & 53 & 10 & 0 & 0 & 1203 & 0 & 908 & 0 & 192 & 33 & 48 & 56 \\
\hline $420-24$ & 15 & 1 & 240 & 0.08 & 0.01 & 3 & 1 & 30 & 1 & 0 & 0 & 760 & 0 & 588 & 0 & 12 & 19 & 4 & 33 \\
\hline $508-12$ & 37 & 1 & 240 & 0.32 & 0.04 & 6 & 3 & 53 & 14 & 0 & 0 & 2383 & 0 & 1829 & 0 & 131 & 54 & 43 & 91 \\
\hline $512-16$ & 34 & 1 & 240 & 0.31 & 0.08 & 7 & 5 & 47 & 15 & 0 & 0 & 2162 & 0 & 1661 & 0 & 231 & 67 & 67 & 101 \\
\hline 5 16-20 & 35 & 2 & 240 & 0.34 & 0.10 & 7 & 8 & 53 & 12 & 0 & 0 & 1910 & 0 & 1488 & 0 & 612 & 79 & 119 & 106 \\
\hline $520-24$ & 59 & 3 & 240 & 0.69 & 0.25 & 7 & 9 & 95 & 29 & 0 & 0 & 2753 & 0 & 2080 & 0 & 1314 & 121 & 237 & 170 \\
\hline $604-08$ & 112 & 3 & 239 & 1.48 & 0.69 & 8 & 9 & 197 & 73 & 0 & 0 & 5035 & 0 & 3678 & 0 & 1658 & 213 & 243 & 307 \\
\hline $608-12$ & 111 & 3 & 107 & 2.40 & 1.26 & 10 & 23 & 233 & 55 & 0 & 0 & 4954 & 0 & 3610 & 0 & 886 & 445 & 170 & 396 \\
\hline
\end{tabular}

Table 7: Algorithmic information for binary objective function.

\begin{tabular}{||c||c||c|c||c|c||c||}
\hline \multicolumn{1}{||c||}{ Day } & \multicolumn{1}{c||}{ Runs } & \multicolumn{2}{c||}{ Optimal (\%) } & \multicolumn{2}{c||}{ Run time (s) } & Speed up \\
& & BM & PC & BM & PC & \\
\hline \hline 26 & 696 & $100 \%$ & $100 \%$ & 0.95 & 0.60 & $1.58 \mathrm{x}$ \\
\hline 27 & 1398 & $100 \%$ & $100 \%$ & 1.41 & 1.40 & $1.01 \mathrm{x}$ \\
\hline 28 & 1362 & $100 \%$ & $100 \%$ & 0.75 & 0.52 & $1.44 \mathrm{x}$ \\
\hline 29 & 1434 & $100 \%$ & $100 \%$ & 0.24 & 0.04 & $6.00 \mathrm{x}$ \\
\hline 30 & 1440 & $100 \%$ & $100 \%$ & 0.32 & 0.09 & $3.56 \mathrm{x}$ \\
\hline
\end{tabular}

Table 8: Aggregate comparisons for 5 days of computations using the binary objective.

\section{Final remarks and future developments.}

The non-compact formulation introduced in this paper for certain job-shop scheduling problems arising in transportation networks favorably compares with the classic big$M$ formulation, in particular on a class of non-convex cost functions which are commonly used in a real-life setting. A merit of the new approach is to open a number of potential research paths.

A first candidate for investigation is the fact that the constraints in the new formulation correspond to basic graph structures (such as paths, cycles and trees). This opens the way for strengthening the formulation through polyhedral work, identifying classes of valid, possibly facet-defining inequalities. Other interesting modeling developments to look into are the integration of this approach with routing, which was not considered in this paper, and the relaxation of the non-decreasing cost function assumption. 


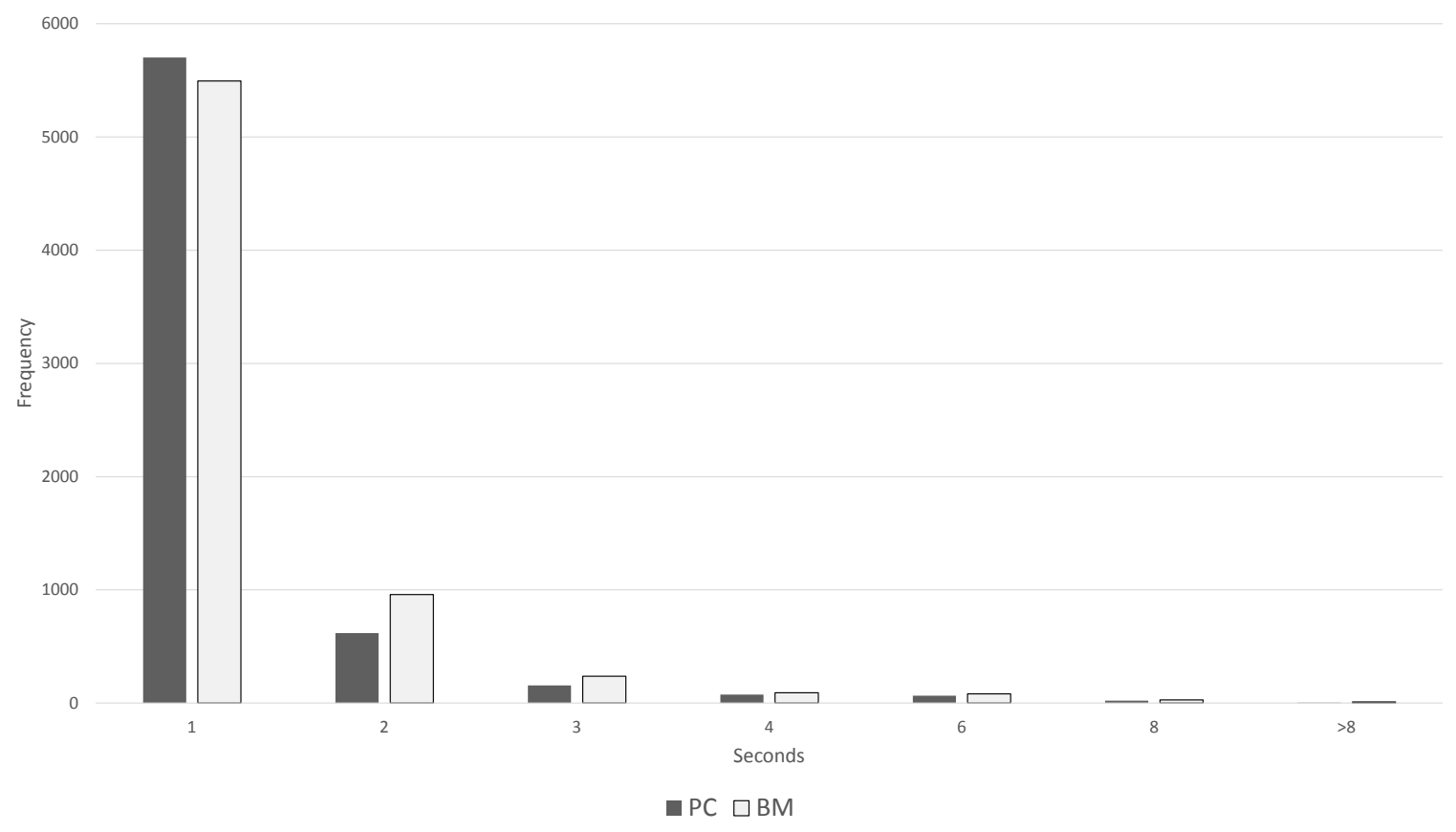

Figure 14: Histogram of the frequency with respect to computational time for instances in Table 7.

From an algorithmic standpoint, a natural enhancement is to separate the the path and cycle constraints during the Branch\&Bound process, rather than only at the end.

Finally, the approach can be extended to tackle other related applications such aircraft scheduling and routing.

On a final note, as mentioned we expect this new formulation to be particularly suited for the class of job-shop scheduling problems that arise in transport traffic management because of their common structure. In particular, the fact that the number of "necessary" disjunctions is limited, typically in the order of the number of operations, and that the objective function is generally associated with the timing of few operations (e.g. the delay of a vehicle at a given location). It seems natural to extend the approach to handle other, similar traffic management problems, such as train delay management, runway scheduling and airport ground movement management. At a later stage, it may be interesting to also investigate its performance on other job-shop scheduling problems in possibly non-transport related application areas.

\section{References}

[1] R.K. Ahuja, T.L. Magnanti, J.B. Orlin, Network Flows, Prentice-Hall, 1993. 


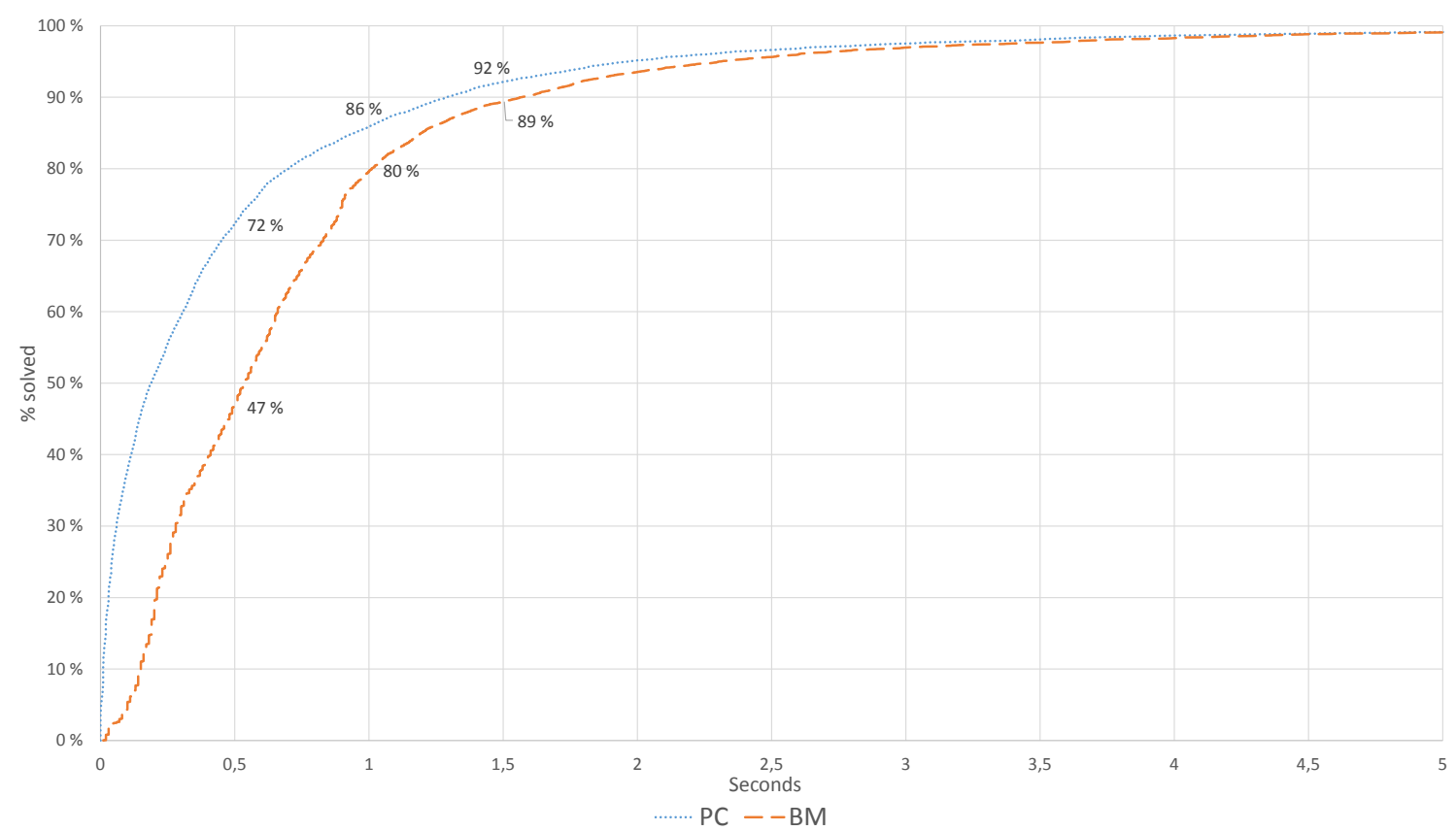

Figure 15: Cumulative distribution of the percentage of instances solved in Table 7 with respect to computation time.

[2] D. Alvras, M.W. Padberg, Linear Optimization and Extensions: Problems and Soluzions. Springer-Verlag, Berlin, Germany, 2001.

[3] E. Balas, Machine sequencing via disjunctive graphs, Operations Research 17 (1969) pp. 941-957.

[4] E. Balas, Facets of the knapsack polytope, Mathematical Programming 8 (1), pp. 146-164, 1975.

[5] E. Balas, Disjunctive programming, Annals of Discrete Mathematics, 5, pp. 3-51, 1979.

[6] J.A. Bennell, M. Mesgarpour, C.N. Potts, Airport runway scheduling, 4OR 9(2) 115-138, 2011.

[7] D. Bertsimas, J. Tsitsiklis, Introduction to linear optimization. Vol. 6. Belmont, MA: Athena Scientific, 1997

[8] P. Bonami, A. Lodi, A. Tramontani, A., S. Wiese, 2015, On mathematical programming with indicator constraints, Mathematical Programming, 151(1), pp. 191-223, 2015 . 
[9] V. Cacchiani, D. Huisman, M. Kidd, L. Kroon, P. Toth, L. Veelenturf, J. Wagenaar, An overview of recovery models and algorithms for real-time railway rescheduling, Transportation Research Part B, 63, pp. 15-37, 2014.

[10] V. Cacchiani, P. Toth Nominal and robust train timetabling problems, European Journal of Operational Research 219, pp 727-737, 2012.

[11] G. Codato, M. Fischetti, Combinatorial Benders' Cuts for Mixed-Integer Linear Programming, Operations Research, 54 (4), pp. 756-766, 2006.

[12] F. Corman, L.Meng, A review of online dynamic models and algorithms for railway traffic management, Intelligent Transportation Systems, IEEE Transactions on 16 (3), pp. 1274-1284, 2015.

[13] G. Desaulniers, J. Desrosiers, M.M. Solomon, eds. Column generation, Vol. 5. Springer Science \& Business Media, 2006.

[14] M. Dyer., L. Wolsey, Formulating the single machine sequencing problem with release dates as a mixed integer program, Discrete Applied Mathematics, no. 26 (2-3), pp. 255-270, 1990.

[15] M. Fischetti, M. Monaci, Using a general-purpose MILP solver for the practical solution of real-time train rescheduling, Tech Rep. DEI, Univ. Of Padova, 2016.

[16] D. Kjenstad, C. Mannino, P. Schittekat, M. Smedsrud, Integrated surface and departure management at airports by optimization, IEEE Xplore Digital Library, 5th International Conference on Modeling, Simulation and Applied Optimization (ICMSAO), Hammamet 2013.

[17] L. Lamorgese, C. Mannino, An exact decomposition approach for the real-time Train Dispatching problem, Operations Research, 63 (1), pp. 48-64, 2015.

[18] L. Lamorgese, C. Mannino, M. Piacentini, Optimal Train Dispatching by Benders'-like reformulation, Transportation Science, published on line (http://pubsonline.informs.org/doi/10.1287/trsc.2015.0605), 2016.

[19] L. Lamorgese, C. Mannino, E. Natvig, An exact micro-macro approach to cyclic and non-cyclic train timetabling, Omega, 72, 59-70, 2017.

[20] C. Mannino, A. Mascis, Real-time Traffic Control in Metro Stations, Operations Research, 57 (4), pp 1026-1039, 2009

[21] H. Marchand, L.A. Wolsey, The 0-1 knapsack problem with a single continuous variable, Mathematical Programming 85 (1), pp. 15-33, 1999.

[22] A. Mascis, D. Pacciarelli, Job shop scheduling with blocking and no-wait constraints, European Journal of Operational Research, 143 (3), pp. 498-517, 2002. 
[23] G.L. Nemhauser, L.A. Wolsey, Integer and Combinatorial Optimization, WileyInterscience, 1999.

[24] M.N. Nielsen, Danish State Railways, presentation at the Dagstuhl seminar on Algorithmic Methods for Optimization in Public Transport, available at http://materials.dagstuhl.de/index.php?semnr=16171 (last access 28.06.2016).

[25] https://www.forskningsradet.no/prosjektbanken/\#/project/NFR/267554/Sprak=en.

[26] M.L. Pinedo, Scheduling: theory, algorithms, and systems, Springer Science \& Business Media, 2012.

[27] Queyranne, M., A.S. Schulz, Polyhedral Approaches to Machine Scheduling, Tech Rep. 408/1994, Technische Universitat Berlin, 1994.

[28] M. Schachtebeck and A. Schöbel, To Wait or Not to Wait - And Who Goes First? Delay Management with Priority Decisions, Transportation Science, 44 (3), pp. 307-321, 2010.

[29] A. Schrijver, Combinatorial Optimization, Springer, 2003.

[30] T. Schlechte, R. Borndörfer, B. Erola, T. Graffagnino, E. Swarat, Micro-macro transformation of railway networks, Journal of Rail Transport Planning \& Management, 1, pp. 38-48, 2011.

[31] T. Vidal, T.G. Crainic, M. Gendreau, C. Prins, Timing problems and algorithms: Time decisions for sequences of activities, Networks, 65(2), pp. 102-128, 2015.

[32] E. Zemel, Lifting the facets of zero-one polytopes, Mathematical Programming 15, pp. 268-277, 1978.

\section{Appendix: proof of Lemma 3.4}

To prove Lemma 3.4 we use the following result by Marchand and Wolsey ([21]):

Lemma 7.1 Let $Y=\left\{(y, \eta) \in\{0,1\}^{|N|} \times \mathbb{R}_{+}^{1}: \sum_{j \in N} a_{j} y_{j} \leq b+\eta\right\}$, where $0<a_{j} \leq b$ for $j \in N$. Let $Y_{0}=\{(y, \eta) \in Y: \eta=0\}$.

If $\sum_{j \in N} \pi_{j} y_{j} \leq \pi_{0}$ with $\pi \geq 0, \sum_{j \in N} \pi_{j}>\pi_{0}>0$ defines a facet of conv $\left(Y_{0}\right)$, then $\sum_{j \in N} \pi_{j} y_{j} \leq \pi_{0}+\frac{\eta}{\beta}$ defines a facet of $\operatorname{conv}(Y)$, where $\beta=\min _{\eta>0} \frac{\eta}{\sigma(\eta)-\pi_{0}}$, $\sigma(\eta)=$ $\max \left\{\sum_{j \in N} \pi_{j} y_{j}: \sum_{j \in N} a_{j} y_{j} \leq b+\eta, y \in\{0,1\}^{|N|}\right\}$.

Proof of Lemma 3.4. We first show that (17) is facet defining for the $\operatorname{conv}\left(Y^{N}\right)$, where

$$
Y^{N}=\left\{(y, \eta) \in\{0,1\}^{|N|} \times \mathbb{R}_{+}^{1}: \sum_{e \in N} M_{e} y_{e} \leq \sum_{e \in N} M_{e}-c(H)+\eta\right\}
$$


If $N=\emptyset$, both (17) and the knapsack defining $Y^{N}$ reduces to $c(H) \leq \eta$ and the lemma follows trivially.

If $N=\{e\}$, the knapsack defining $Y^{N}$ reduces to $M_{e} y_{e} \leq M_{e}-c(H)+\eta$. It is not difficult to see that $Y^{N}=\left\{\left\{\left(y_{e}=0, \eta\right): \eta \geq 0\right\} \cup\left\{\left(y_{e}=1, \eta\right): \eta \geq c(H)\right\}\right\}$, for which $\operatorname{conv}\left(Y^{N}\right)$ is defined by the trivial facets $y_{e} \geq 0, y_{e} \leq 1$ and $c(H) y_{e} \leq \eta$, the latter coinciding with (17).

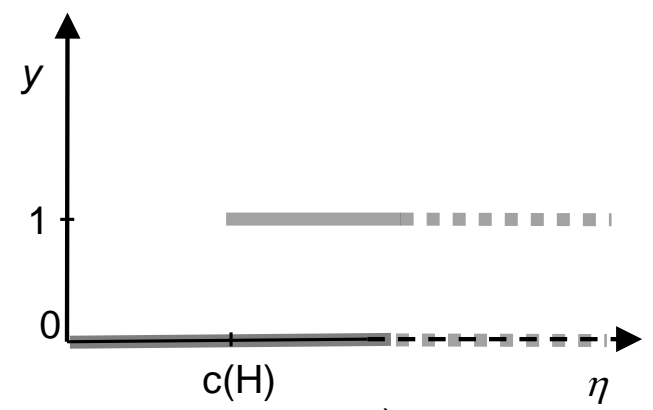

a)

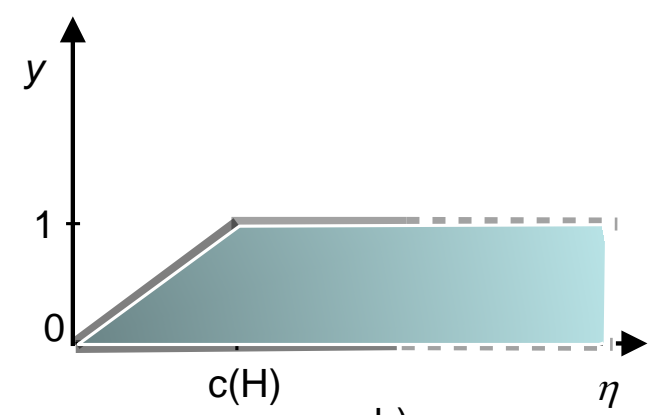

b)
Figure 16: a) $Y^{N}$ for $|N|=1$ (in gray)
b) $\operatorname{conv}\left(Y^{N}\right)$

The remaining case is $|N| \geq 2$, for which we will use the same notation of Lemma 7.1. For $\eta=0$, the knapsack defining $Y^{N}$ reduces to the following binary knapsack:

$$
\sum_{e \in N} M_{e} y_{e} \leq \sum_{e \in N} M_{e}-c(H)
$$

Observe that, for $e \in N$, we have $0<M_{e} \leq \sum_{e \in N} M_{e}-c(H)=b$ and the conditions of Lemma 7.1 are satisfied. The only (minimal) cover of the above knapsack constraint is $N$. Therefore, the cover inequality

$$
\sum_{e \in N} y_{e} \leq|N|-1
$$

is facet defining for the polyhedron $\operatorname{conv}\left(Y_{0}^{N}\right)$.

So, we have

$$
\sigma(\eta)=\max \left\{\sum_{e \in N} y_{e}: \sum_{e \in N} M_{e} y_{e} \leq \sum_{e \in N} M_{e}-c(H)+\eta\right\}= \begin{cases}|N|-1, & 0 \leq \eta<c(H) \\ |N|, & c(H) \leq \eta\end{cases}
$$

and thus 


$$
\beta=\min _{\eta>0} \frac{\eta}{\sigma(\eta)-(|N|-1)}=\min _{\eta>0}\left\{\begin{array}{ll}
\frac{\eta}{|N|-1-(|N|-1)}, & 0 \leq \eta<c(H) \\
\frac{\eta}{|N|-(|N|-1)}, & c(H) \leq \eta
\end{array}=c(H) .\right.
$$

Having computed $\beta$ we can substitute in the expression in the statement of Lemma 7.1 and the constraint

$$
\sum_{e \in N} y_{e} \leq|N|-1+\frac{\eta}{c(H)}
$$

is facet defining for $\operatorname{conv}\left(Y^{N}\right)$. To complete the proof we need to show that $(27)$ is also facet defining for $\operatorname{conv}\left(Y^{H}\right)$, as $N$ may be a proper subset of $F \cap H$. But since $M_{e}=0$ for $e \in(F \cap H) \backslash N$, this derives from simple lifting results (see [32]). 